\title{
LncRNAs in polyploid cotton interspecific hybrids are derived from transposon neofunctionalization
}

\author{
Ting Zhao ${ }^{1,2}$, Xiaoyuan Tao², Shouli Feng ${ }^{1}$, Luyao Wang ${ }^{1}$, Hui Hong ${ }^{3}$, Wei Ma², Guandong Shang ${ }^{1}$, Shisong Guo', \\ Yuxin $\mathrm{He}^{1}$, Baoliang Zhou ${ }^{{ }^{*}}$ and Xueying Guan ${ }^{1,2^{*}}$
}

\begin{abstract}
Background: Interspecific hybridization and whole genome duplication are driving forces of genomic and organism diversification. But the effect of interspecific hybridization and whole genome duplication on the non-coding portion of the genome in particular remains largely unknown. In this study, we examine the profile of long non-coding RNAs (IncRNAs), comparing them with that of coding genes in allotetraploid cotton (Gossypium hirsutum), its putative diploid ancestors (G. arboreum; G. raimondii), and an $F_{1}$ hybrid (G. arboreum $\times$ G. raimondii, AD).

Results: We find that most IncRNAs (80\%) that were allelic expressed in the allotetraploid genome. Moreover, the genome shock of hybridization reprograms the non-coding transcriptome in the $F_{1}$ hybrid. Interestingly, the activated IncRNAs are predominantly transcribed from demethylated TE regions, especially from long interspersed nuclear elements (LINES). The DNA methylation dynamics in the interspecies hybridization are predominantly associated with the drastic expression variation of IncRNAs. Similar trends of IncRNA bursting are also observed in the progress of polyploidization. Additionally, we find that a representative novel IncRNA XLOC_409583 activated after polyploidization from a LINE in the A subgenome of allotetraploid cotton was involved in control of cotton seedling height.

Conclusion: Our results reveal that the processes of hybridization and polyploidization enable the neofunctionalization of IncRNA transcripts, acting as important sources of increased plasticity for plants.
\end{abstract}

\section{Introduction}

Interspecific hybridization and polyploidization are known as intrinsic powers behind genome evolution. Polyploidization, also known as whole genome duplication (WGD), is commonly observed in the evolution of angiosperm plants [1]. Polyploidy, especially allopolyploidy, stabilizes the vigor traits created by hybridization. The genomic interactions in hybrids and polyploids trigger a rapid and extensive reprogrammed response, associated with dramatic changes in the epigenetic modifications involved with, but not limited to, the following: DNA methylation, siRNAs, transposable elements (TEs), and histone modification [2, 3]. The integrative results of these genome-wide modifications

\footnotetext{
* Correspondence: baoliangzhou@njau.edu.cn; xueyingguan@njau.edu.cn ${ }^{1}$ State Key Laboratory of Crop Genetics and Germplasm Enhancement, Cotton Hybrid R \& D Engineering Center (the Ministry of Education), College of Agriculture, Nanjing Agricultural University, Nanjing 210095, Jiangsu, China Full list of author information is available at the end of the article
}

lead to expression changes in about $20-50 \%$ of mRNA, which is the proposed molecular basis for the vigor of hybridization and polyploidization $[4,5]$.

Small interfering RNAs (siRNAs), especially those derived from TEs, can suppress mobile element activity via RNA-directed DNA methylation (RdDM) [6-8]. In hybrids of Arabidopsis thaliana, rice, and maize, expression levels of siRNAs changed dramatically upon polyploidization [9-11]. DNA methylation changes also coincided with activation of TEs in analysis of intraspecific hybrids of $A$. thaliana, rice, and maize [12-16]. However, the impact of hybridization and polyploidization on the lncRNA of whole genomes remains largely unknown.

Long noncoding RNAs (lncRNAs) are typically transcribed from the intergenic regions of the genome, while some lncRNAs originate from the antisense strands of coding genes [17]. In the last few years, lncRNAs have been widely identified in both animal and plant genomes [18-22]. In animal genomes, lncRNAs are associated 
with X chromosome inactivation [23], disease development [24], etc. Epigenetic modifications on lncRNA are reported to play critical roles on its expression and function. More than 1000 lncRNA genes are found to be hypo-methylated in cancer cell lines recurrently [25]. In plants, lncRNAs are reported to play critical roles in multiple regulation functions, such as developmental regulation [26-28] and both biotic and abiotic stress responses [26, 29-31]. Although the many functions of lncRNA have gradually been elucidated, the exact origin of lncRNA is still obscure.

According to previous genome-wide investigations, IncRNA transcriptomes appeared unique to each species $[32,33]$. For example, mRNA similarity between genomes of human and mouse is $92 \%$, but the lncRNA similarity between them is as low as $35 \%$ [34]. Less than $6 \%$ of zebrafish lincRNAs (long intergenic RNAs) have any detectable sequence similarity to human and mouse lincRNAs [35]. In the comparison of 16 vertebrate species and the sea urchin, $>70 \%$ of lincRNAs cannot be traced to homologs in species that diverged $>50$ million years ago [36]. Similar trends have been observed in plant species. For example, less than $0.4 \%$ of predicted lncRNAs were reported to present in two different tomato species [37]. These data suggest that a genome can generate a large amount of novel lncRNAs efficiently when a new species comes into being. The origin of these species-specific lncRNAs is still unknown.

The latest studies have reported that transposon elements (TEs) might be involved with lncRNA origin and diversification [38-40]. For example, Xist originated from a coding gene, $\operatorname{Ln} \times 3$, with accumulated TEs in its exons [41]. We previously found that an NAT (natural antisense transcript) originating from a locus on the coding gene GhMML3 is associated with TE insertion. This NAT caused the fuzzless seed mutant of $N_{1}$ by suppressing GhMML3 expression [42]. TEs are abundant in advanced organisms, especially in plants. For example, TEs comprise $80 \%$ of the maize genome and $65 \%$ of the cotton genome [43-46]. TEs can be classified as retrotransposons and DNA transposons, each with diverse patterns in sequence and activity [47]. We still do not know which type of TE is related to lncRNA origin.

The behavior of IncRNAs during hybridization and polyploidization provides an important clue to the origin of IncRNA. Here we utilized a simplified model of cotton hybridization and polyploidization to study the origin of IncRNA. Cotton is not only a source of natural and renewable fiber for textiles, but also a fine model for heterosis studies. Regarding evolutionary lineage, upland cotton (Gossypium hirsutum, (AADD) ${ }_{1}$, Gh), an allotetraploid species, was formed after the hybridization and polyploidization of its two closest extant progenitors, $G$. herbaceum ( $\mathrm{A}_{1}$ genome) or $\mathrm{G}$. arboreum ( $\mathrm{A}_{2}$ genome), and $G$. raimondii ( $\mathrm{D}_{5}$ genome), about 1-1.5 million years ago (MYA) [44, 48]. The two diploid progenitors diverged 6-6.3 MYA [44, 48]. We crossed G. arboreum $(\mathrm{Ga})$ accession from Shixiya with $G$. raimondii $(\mathrm{Gr})$, generating an $\mathrm{F}_{1}$ hybrid. Using $\mathrm{Ga}, \mathrm{Gr},(\mathrm{Ga} \times \mathrm{Gr}) \mathrm{F}_{1}$, and $\mathrm{Gh}$ (accession Texas Marker-1 (TM-1)), we constructed a system to mimic the evolution of Gossypium spp. from diploid to allotetraploid. To determine the origin and behavior of lncRNAs in plant genome evolution, we used methods of interspecific comparative genomics, after identifying 1:1 lncRNA orthologs between species. Based on our integrative analysis of lncRNA sequencing, small RNA sequencing, ChIP-Seq, and DNA methylation data, our results suggested that LINEs arising from TEs play a crucial role in the origin of lncRNAs.

\section{Results}

\section{The Gossypium lineage-specific IncRNA transcriptome}

We generated ribo-depleted strand-specific RNA-seq libraries of Gossypium hirsutum, the ancestors G. arboreum and G. raimondii, and the $\mathrm{F}_{1}$ hybrid using leaf and ovule tissues (Fig. 1a, Additional file 1: Table S1) [49]. Ultimately 8514 lncRNAs in Gh, 4107 in Ga, 2767 in Gr, and 8126 in $F_{1}$ were obtained respectively (Additional file 1: Table S2). Among the predicted lncRNAs, 90\% were lincRNAs (Additional file 1: Table S3). The genomic features of these lncRNAs were similar to those identified in previous studies, including low CG content, fewer exons, and lower expression levels compared to protein-coding genes (PCGs) (Additional file 2: Figure S1 A-C) $[20,50]$. Approximately $60 \%$ of lncRNAs were closed to PCGs $(<5 \mathrm{~kb})$ (Additional file 2: Figure S1D, E). Two thirds of the lncRNAs were overlapped with repetitive TEs (Additional file 2: Figure S1F).

For comparative genome analysis, the genome sequences of Arabidopsis thaliana, Oryza sativa, and Theobroma cacao were selected to test conservation of lncRNA. Less than $3.83 \%(n=590)$ of lncRNAs showed homologous sites in A. thaliana and O. sativa, from which the cotton genome diverged 87 and 115 million years ago (MYA) [51], respectively. But the primary sequences of most $\operatorname{lncRNAs}(86 \%, n=13,282)$ were common to all three cotton genomes (Fig. 1b, Additional file 2: Figure S2A), which diverged $\sim 2-8$ MYA [44, 48]. A small portion of IncRNAs (12.43\%) had homologs in Theobroma cacao, in contrast to the much greater number of PCG homologs $(69.25 \%$ homologs in T. cacao) (Additional file 2: Figure S2B). The above data confirmed that cotton IncRNAs are predominantly Gossypium lineage-specific.

To obtain a reliable cross-species comparison, the lncRNA loci were classified into syntenic and non-syntenic groups based on their chromosomal locations (Fig. 1c, d, details in method). We first defined the 

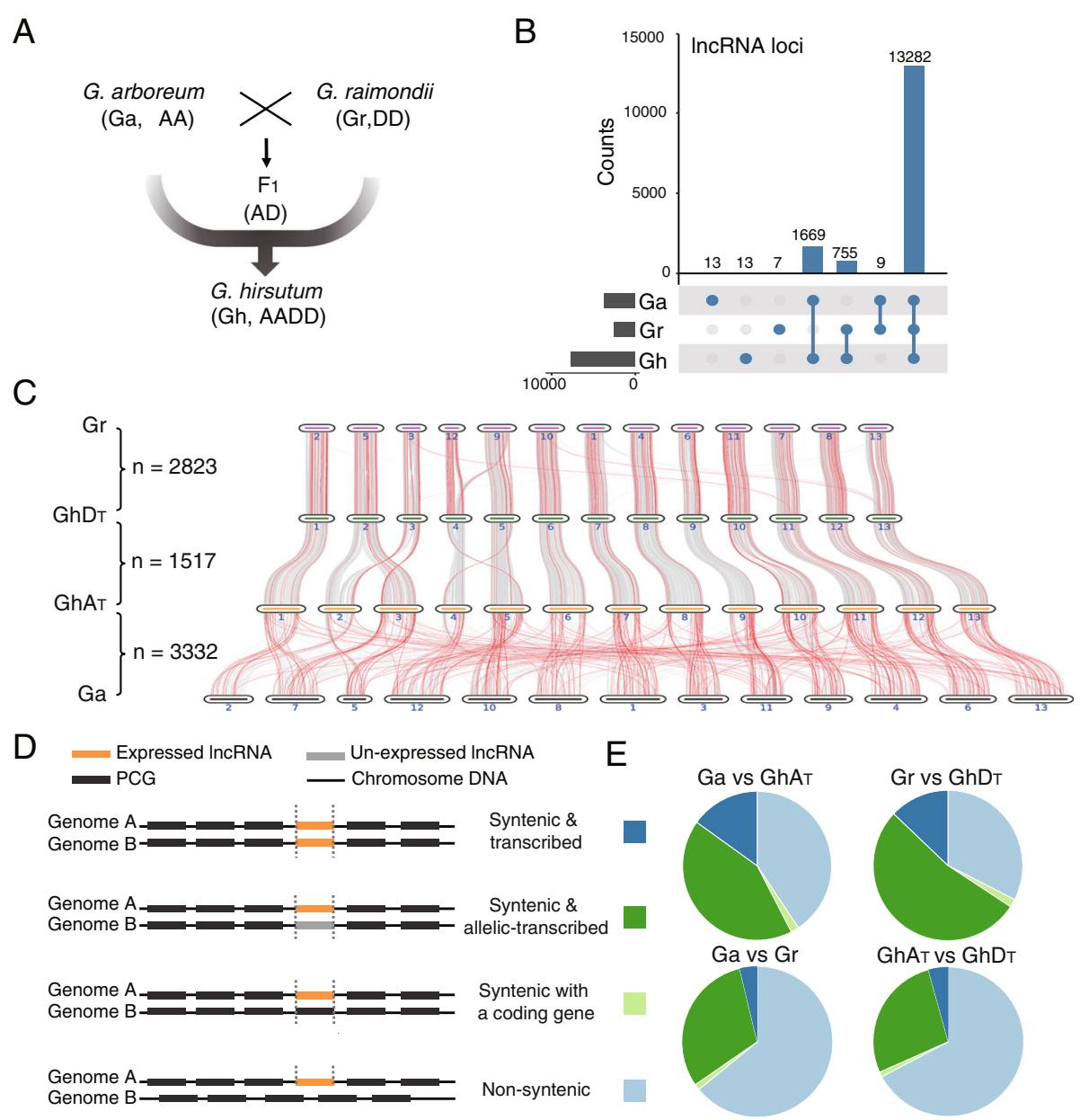

Fig. 1 Lineage-specific and allelic-transcribed cotton IncRNA. a Schematic model for the diploid species (Gossypium arboreum, Ga; G. raimondii, Gr; $F_{1}$; and G. hirsutum, Gh), $F_{1}$ hybrid, and the allotetraploid species. b Histogram showed the IncRNA homologous loci distribution in Ga, Gr, and Gh. $n$ represents the number of IncRNA loci. c Genome-wide identification of syntenic blocks between Ga, Gr, and Gh. $n$ represents syntenic IncRNA in the comparison of different subgenomes. Gray lines represent protein-coding genes (PCGs). Red lines represent IncRNAs. $\mathbf{d}$ Schematic chart showing the IncRNA homologs classified according to syntenic and expressive pattern. e Pie chart showing the number of each classification from panel e

syntenic lincRNA loci, which represent one-to-one homology with lncRNA loci (Fig. 1d, Additional file 1: Table S4). Syntenic groups were then further categorized into three subgroups: (1) syntenic and transcribed (ST), (2) syntenic and allelic-transcribed (SA), and (3) syntenic to a PCG (Fig. 1d, e). NATs and intronic RNAs were excluded from this analysis because of their partial overlap with PCGs. The sequence similarity of ST IncRNA (mean $85.71 \%$ ) was much lower than that of mRNA (mean 96.60\%) (Additional file 2: Figure S3). The SA category constituted the majority $(71.67-86.00 \%)$ of syntenic lncRNA loci in all four comparisons (Fig. 1e). Importantly, the SA lncRNAs comprised over 80\% (1269 out of 1517) of syntenic homologous lncRNA loci (Fig. 1e) in the comparison between $\mathrm{GhA}_{\mathrm{T}}$ (a sub-genome of the allotetraploid (AADD) $)_{1}$ genome) and
$\mathrm{GhD}_{\mathrm{T}}$, based on data from identical genome and tissues. The above data further confirmed that expression of lncRNAs are predominantly species-specific.

\section{LncRNAs are reprogramed in synthetic interspecies $F_{1}$}

Next, we asked whether the genome-specific patterns of lncRNAs were formed in the early stage of hybridization. A comparison of assembled lncRNAs between $F_{1}$ and the parent genome was conducted. We identified in total 8514 lncRNA in $\mathrm{F}_{1}$; only $29.47 \%$ ( $n=2,395$, Fig. $2 \mathrm{a}$ ) were overlapped with those of its parents. Furthermore, an in silico hybrid was constructed by mixing the diploid parental RNA-seq data in a ratio of $1: 1$, to reflect the accumulated gene expression divergence of the two parents without the impact of hybridization [52]. The lncRNA annotation files of $\mathrm{Ga}, \mathrm{Gr}$, and $\mathrm{F}_{1}$ were merged using 
A

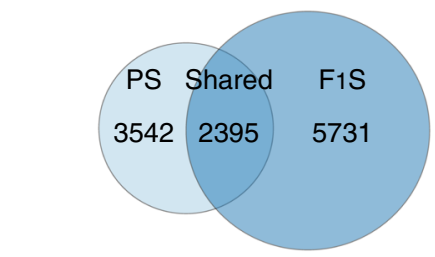

C

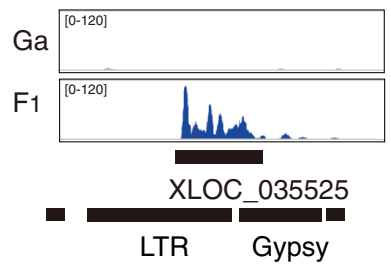

D

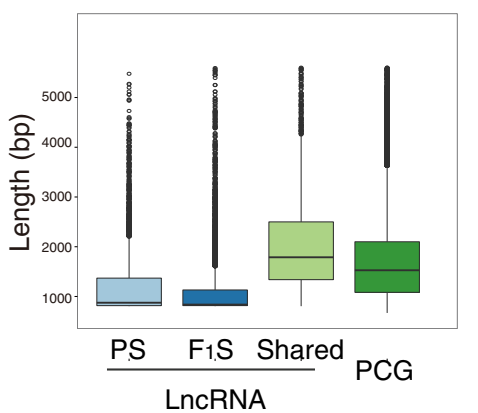

B

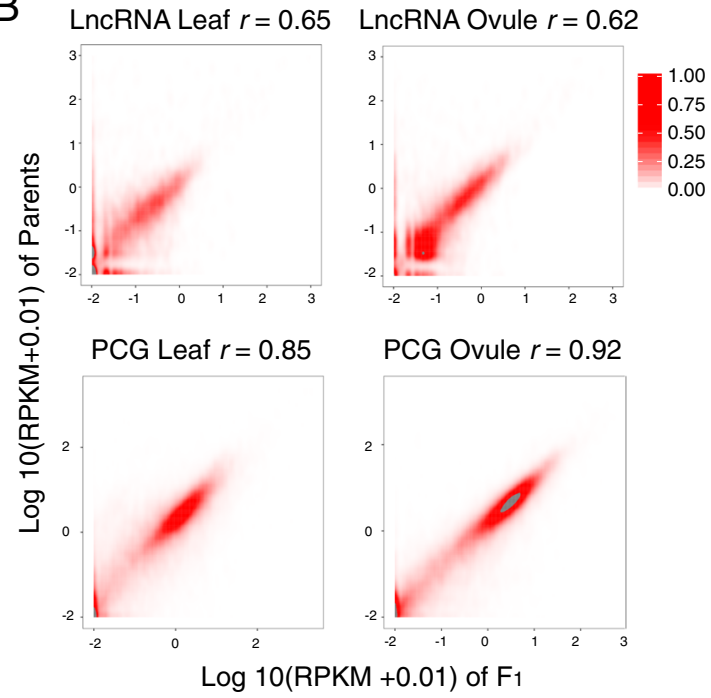

$E$

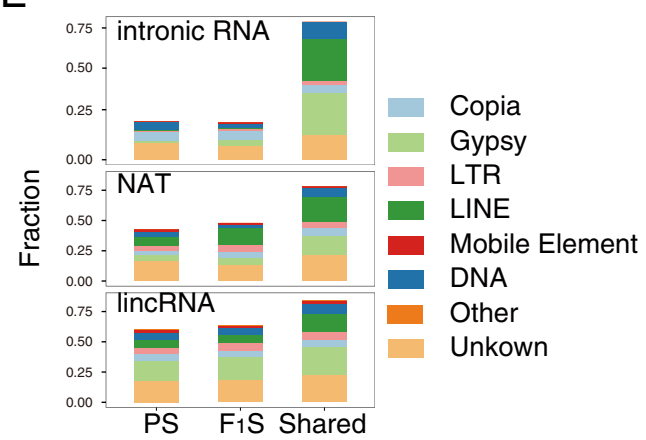

Fig. 2 LncRNA reprogrammed in synthetic $F_{1}$ a Venn diagram showing overlapped IncRNA assembled in parents and $F_{1}$. LncRNAs were grouped into three catalogs. Parent-specific (PS), $F_{1}$-specific $\left(F_{1} S\right)$, and shared IncRNA. b Dot plot representing the expression of IncRNA and PCGs in $F_{1}$ compared to parents. $r$ is the Spearman correlation. c Stacking diagram illustrating a TE-overlapped IncRNA allelic-transcribed in $F_{1}$ hybrid. d Length distribution of parent-specific, $F_{1}$-specific, and shared InCRNA in $F_{1}$ and parents. PCGs are the control group. e Distribution of parentspecific, $F_{1}$-specific, and shared IncRNA overlapped with transposons

cuffmerge [53]. In stark contrast to PCGs, we found lncRNAs exhibited more expression variance $(r$ value by Spearman correlation, $0.62-0.65$ for lncRNA versus 0.85-0.92 for mRNA) (Fig. 2b). For example, lncRNA XLOC_035525 was a novel transcript from the non-coding region of Ga stimulated in hybrid (Fig. 2c). Therefore, we observed a burst of lncRNA transcription in the interspecific hybridization of $F_{1}$.

To exclude the possibility that the IncRNA burst was triggered by inbreeding, two near-isogenic lines (NILs) from inbred lines of cotton, upland cotton (Gh, accession Zhong12), and Zhong12 GL (a dominant, glandless line produced by multiple generations of backcrossing with Zhong12) were selected as control group. These NILs share identical genetic backgrounds. Using the same pipeline we had constructed earlier, a total of 4615 lncRNAs from the control group were identified (Additional file 1: Table S2). In the comparison between Zhong12 and Zhong12 GL, only $1.71 \%$ (73 out of 4281 ) of lncRNAs were differentially expressed $(p<0.05)$. In contrast to the lncRNA expression pattern in the NILs, the in silico parents and $F_{1}$ exhibited a total of $34.75 \%$ (1999 out of 5752) differentially expressed lncRNA loci in leaves and 50.00\% (2594 out of 5184) in ovules. The global changes in the lncRNAs of $F_{1}$ suggest that interspecies hybridization stimulated a reprogramming of transcription on the non-coding region of the genome.

\section{The conserved IncRNAs are overlapped with TEs}

To investigate the effective factors affecting lncRNA preservation in polyploidization, we anchored our analysis on the $F_{1}$ genome. The shared lncRNAs in $F_{1}$ and parents tended to have long transcripts and were overlapped with TEs (Fig. 2d, e). The proportions of $F_{1}$-specific $\left(\mathrm{F}_{1} \mathrm{~S}\right)$ and parent-specific (PS) lncRNAs overlapping with TEs were $63.49 \%$ and $60.41 \%$, respectively, while in conserved IncRNAs, this proportion was as high as $84.47 \%$. This phenomenon was also observed in intronic 
RNA and NATs (Fig. 2e). Considering all of these results, we hypothesized that TE was involved with lncRNA retention and burst in hybridization.

LncRNAs are constrained on LINE and Gypsy-overlapped loci Previous comparative studies of human, mouse, and zebrafish genomes indicated that non-TE lncRNAs might suffer relatively high evolutionary constraint than TE-derived lncRNAs do [40]. TE might contribute to the evolution of IncRNA in both the short (i.e., interspecies hybrid) and long term (i.e., polyploidization).

TEs can be classified into two groups in general, DNA transposons and retrotransposons [47]. Retrotransposons can be further classified into LINE, SINE, and LTR according to structure variation [47]. We assessed the distribution of each type of TEs on lncRNA loci using PCGs as control. According to the analysis with BEDTools intersect [54], the lncRNA loci contained significantly more TE segments at the upstream 2000 bp regions, exon sequences, intron sequences, and downstream 2000 bp regions than did PCGs (Fig. 3a). This striking trend was in agreement with the reported observations of human, mouse, and zebrafish vertebrate genomes [40]. The Gypsy showed the largest proportion of lncRNA-overlapped TEs due to its largest share of TEs in the cotton genome (Fig. 3a). However, LINEs comprised $\sim 40 \%$ of IncRNA-overlapped TEs on exons in each cotton species, although this type of TE only occupied $0.81-1.65 \%$ of the cotton genome as a whole (Fig. 3a). This indicated that LINE might distinctively impact the lncRNAs.

The association of TEs and lncRNA expression in the ST and SA groups were examined. The comparisons were conducted between the presence frequency of TEs in the ST and SA groups. The distribution of LINEs was skewed toward ST lncRNAs, while Gypsy was significantly enriched in the SA lncRNAs (Fisher's exact test, typical $p<0.01$ ) (Fig. 3b). The correlation coefficient of TE-overlapped lncRNA expression levels between the parents and $F_{1}$ was calculated. Surprisingly, an even stronger correlation coefficient was observed for LINE-overlapped lncRNAs compared to Gypsy-overlapped lncRNAs, both in ovule (LINE $r=0.92$; Gypsy $r=0.66$ ) and leaf tissues (LINE $r=0.87$; Gypsy $r=0.63$ ) (Fig. 3c and Additional file 2: Figure S4). These association test results suggested that
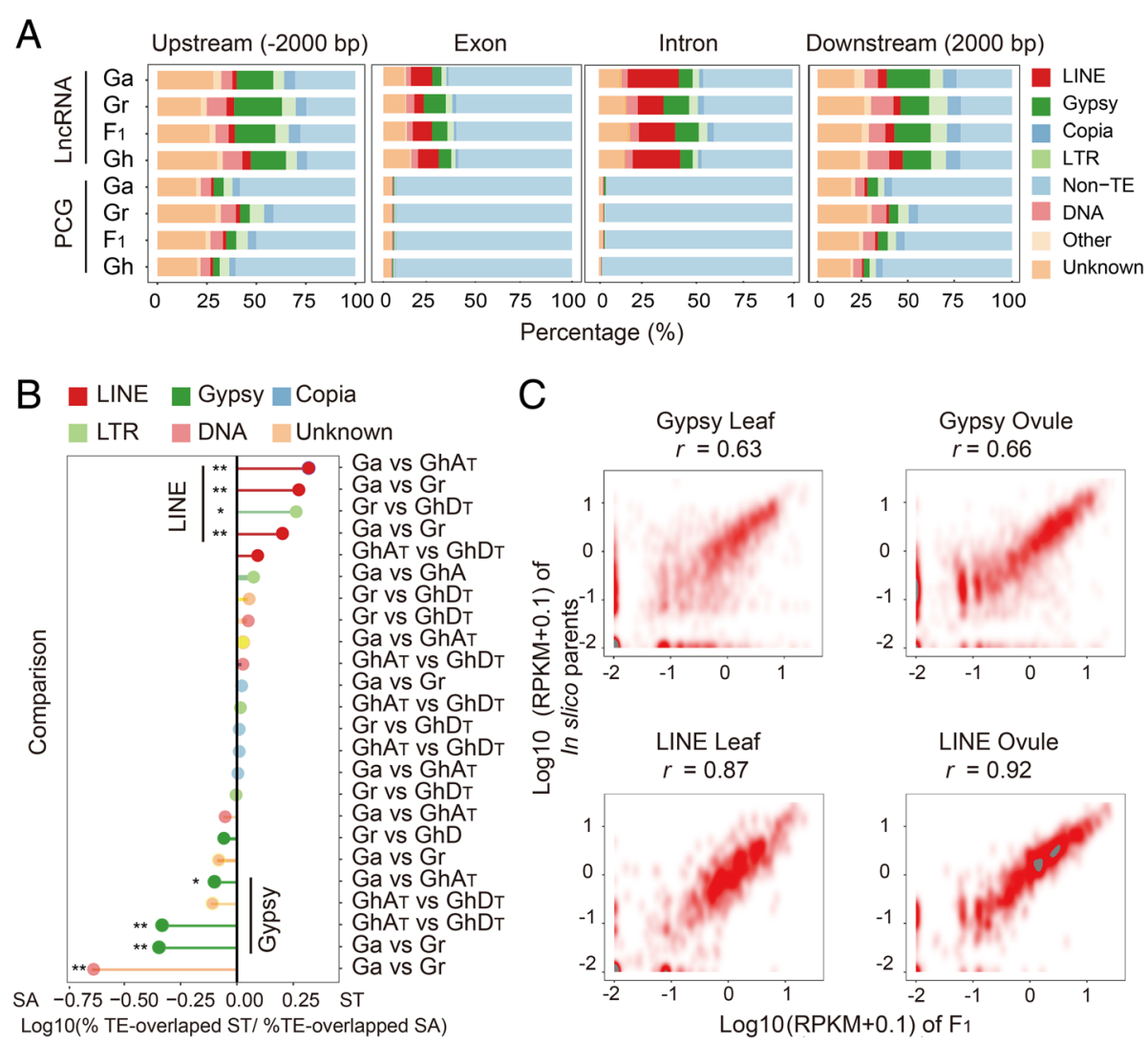

Fig. 3 The impacts of LINE and Gypsy on IncRNA expressions in genome shock. a Distribution of transposons in different gene/IncRNA features. b The ratio of the proportion of TE-overlapped ST IncRNA and TE-overlapped SA IncRNA. Frequencies of many specific TE families in ST and SA IncRNA differ significantly (based on Fisher's exact test). ST IncRNA are above 0 on the $X$ axis, and SA IncRNA are below 0 . c Dot plot representing the expression correlation of Gypsy- and LINE-overlapped IncRNAs in $F_{1}$, compared to the parents. $r$ is the Spearman correlation 
TEs in the categories of LINE and Gypsy represented distinct functional pattern in genome shock.

\section{LncRNAs are transcribed from siRNA-depleted LINEs/TEs}

TEs are generally considered to be major recruiters of epigenetic modifications, such as siRNA and DNA methylation [55-58]. We found that more TEs were overlapped with lncRNAs than PCGs (Fig. 3a). To define the relationship between epigenetic modifications on lncRNAs and TEs, we performed deep small-RNA sequencing for the leaf and ovule tissue of $F_{1}$ (Additional file 1: Table S5). A total of 4.84 billion siRNA reads were obtained after filtering out tasiRNA, microRNA, and snoRNA (Additional file 1: Table S6). Most siRNAs were mapped to TE regions (on average 61.8\%) (Fig. 4a), while PCGs and lncRNA-associated siRNAs occupied $9.89 \%$ and $4.14 \%$ of the total siRNAs respectively. As shown in Fig. 4b, lncRNA Ga_XLOC_435840 was a representative TE-overlapped IncRNA locus that generated
siRNA covering both TE and non-TE regions (Fig. 4b).

Similar to findings in model plant genomes [59]), cotton siRNAs were abundantly enriched in TE bodies, but less in the upstream and downstream of TE bodies (Fig. 4c, Additional file 1: Figure S5). For the PCGs, sRNAs were enriched in the upstream and downstream regions of the gene body and less in the gene body (Fig. 4c, Additional file 1: Figure S5). Meanwhile, the siRNA distribution pattern on lncRNA loci was distinctive from that on both TEs and PCGs (Fig. 4c, Additional file 1: Figure S5). Compared to common TEs, lncRNA-associated TEs were covered by less siRNA (Fig. 4c, Additional file 1: Figure S5).

SiRNAs are known to suppress TE activity via siRNA-directed DNA methylation (RdDM) pathway in plant genomes. Accordingly, we predicted that the expression of TE-overlapped lncRNA could be affected by siRNA. Since LINE-overlapped IncRNAs were more

\section{A}

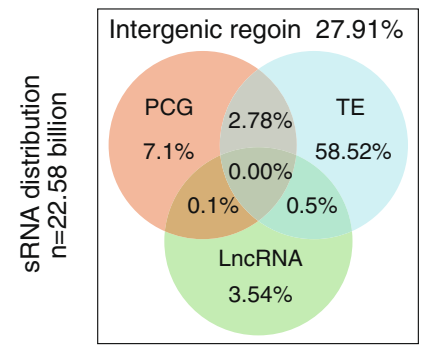

C

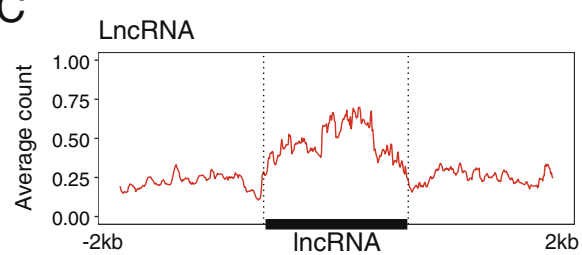

LncRNA-overlapped TE

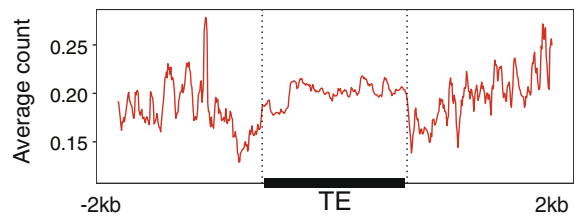

LncRNA(Gypsy/LINE)-overlaped TE

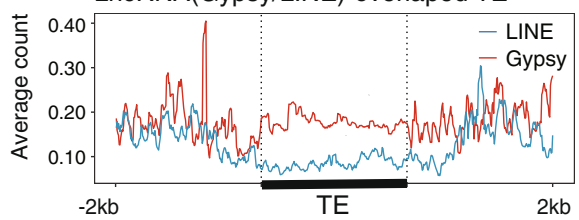

B
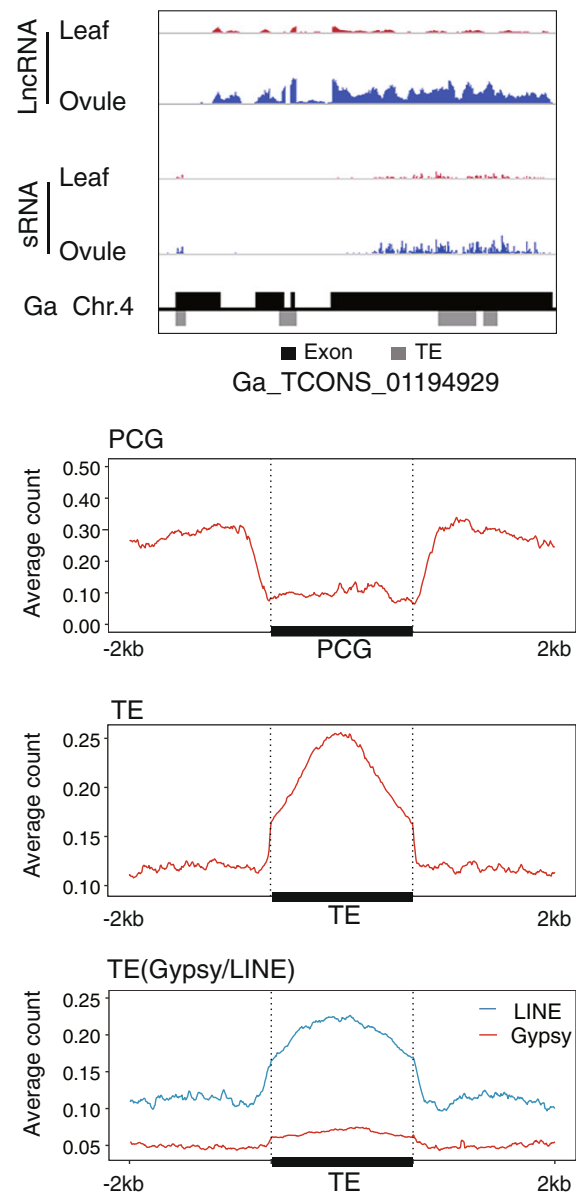

Fig. 4 LncRNAs were transcribed from siRNA-depleted LINEs/TEs. a Venn diagram showing the sRNA distribution in PCG, IncRNA, and TE regions. b Stacking diagram illustrating a TE-overlapped IncRNA transcription and sRNA. c sRNA distribution pattern on IncRNA, mRNA, IncRNA-overlapped TE, and TE regions. Gypsy and LINE types of TE were selected in order to compare sRNA distribution on IncRNA-associated TE and genome-wide TE 
stably transcribed compared to Gypsy-overlapped lncRNAs (Fig. 3c, Additional file 1: Figure S5), we hypothesized that siRNA distribution pattern on lncRNA-overlapped LINE and Gypsy might be different. To test this, we examined the distribution density of siRNA over the Gypsy and LINEs that overlapped with lncRNA regions respectively. As expected, the mapping densities of siRNAs in the transcribed regions of LINEs were much lower than those in Gypsy (Fig. 4c, Additional file 1: Figure S5). Twenty-four nucleotides and $21 \mathrm{nt}$ siRNA were both enriched on IncRNA with similar pattern during the genomic shock (Additional file 1: Figures S6 and S7).

\section{LncRNAs were primarily transcribed from demethylated LINEs/TEs}

To validate the effects of siRNA on the activity of lncRNA and IncRNA-overlapped TE, we further compared the DNA methylation pattern on IncRNAs with PCGs and TEs in general using publicly available bisulfite sequencing (BS-seq) data of $\mathrm{Ga}, \mathrm{Gr}, \mathrm{F}_{1}$, and $\mathrm{Gh}$ [60]. CG methylation accounted for the majority of the 68,166 DMRs in hybridization (CG 63.66\%, CHG $25.33 \%, \mathrm{CHH}, 11.02 \%)$, which was consistent with reports of Arabidopsis and bean genomes [61, 62]. To dissect the DNA methylation dynamics in hybridization, we selected to show the pattern on lncRNAs and PCGs in $\mathrm{F}_{1}$. The CHG and $\mathrm{CHH}$ levels on the IncRNA body region were higher than that in PCGs (Fig. 5a). But for the IncRNA-overlapped TEs, the CG and CHG levels were much less on the TE body (Fig. 5b). Strikingly, the lncRNA-overlapped LINE showed low methylation level on all three contents (Fig. 5c). This trend was in line with the siRNA distribution patterns shown in Fig. 4c. Subsequently, we speculated the DNA methylation changes might impact the lncRNA activity in hybrid.

A dramatic DNA methylation change has been reported in the intraspecific hybrids of A thaliana [62], rice [14], maize [13], and cotton [60], and CG methylation makes the greatest contribution to genome-wide DNA methylation changes in hybrids [13, 15, 60, 62]. In addition, Wang et al. [50] found that lncRNA could be induced by zebularine, a DNA methylation inhibitor, in cotton ovules. In light of this, we wanted to describe in detail the impact of certain methylation changes on lncRNA loci in hybridization. To address this question, we performed further association tests on the differentially methylated regions (DMRs) between the $\mathrm{F}_{1}$ hybrid and its parents ( $\mathrm{Ga}$ and $\mathrm{Gr}$ ). Conversely, the portion of DMRs arising from CG methylation was significantly lower on lncRNA loci (40\%) in $F_{1}$ compared to the total DMRs (64\%). More specifically, the portion of DMR arising from $\mathrm{CHH}$ methylation increased from $11 \%$ of the total level to $22 \%$ on the $F_{1}$ upregulated loci (Fig. 6a). Furthermore, we found that the DMR resulting from $\mathrm{CHG}$ and $\mathrm{CHH}$ in $\mathrm{F}_{1}$ lncRNA loci was predominantly hypo-methylated (Fig. 6b). These data implied that DNA demethylation was in fact active on activated lncRNA loci in the $\mathrm{F}_{1}$ hybrid genome.
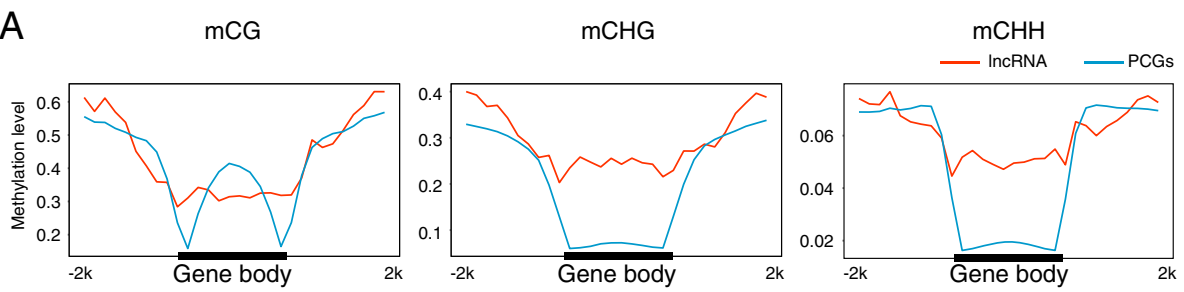

$\mathrm{B}$
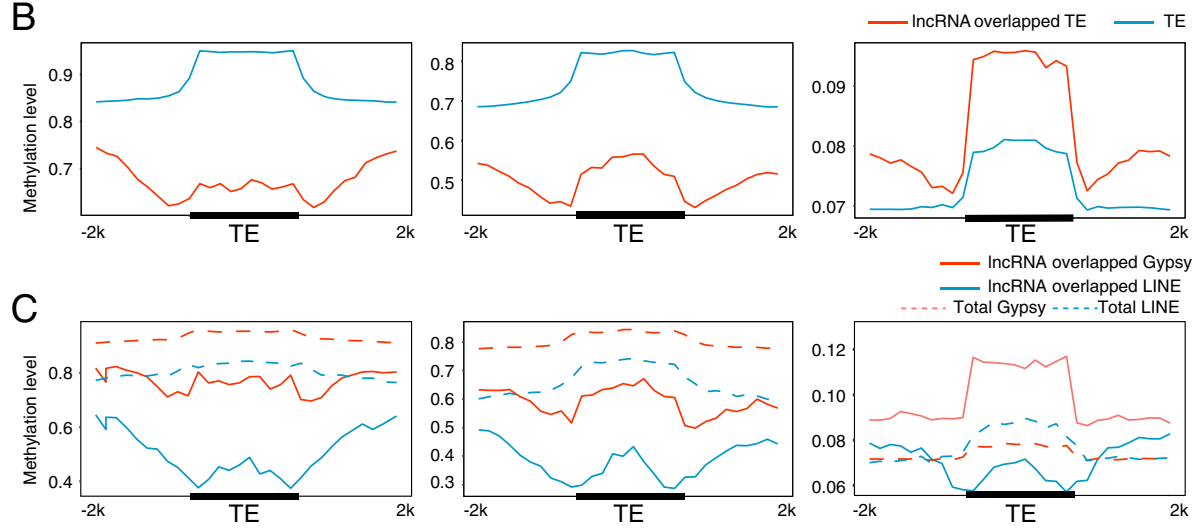

Fig. 5 LncRNA were transcribed from demethylated TEs. Density plots of DNA methylation (CG, CHG, and CHH) profiles on IncRNA versus PCG (a), TE versus IncRNA-overlapped TE (b), and IncRNA-overlapped Gypsy versus IncRNA-overlapped LINE (c) 
A
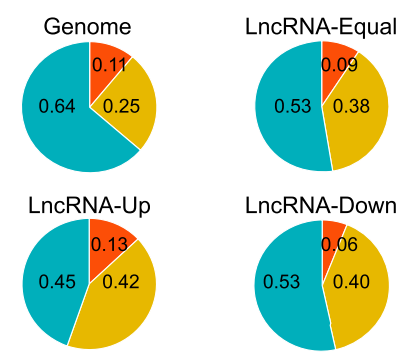

C

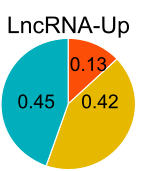

DMR Distribution
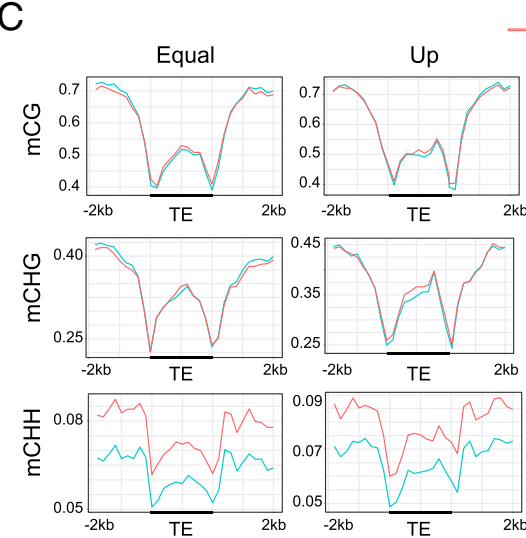

D

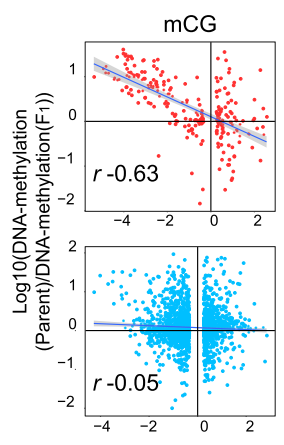

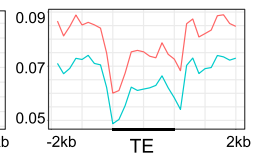
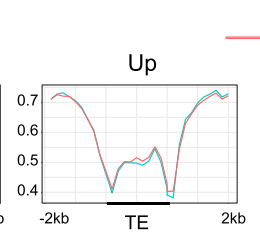

$2 \mathrm{~kb}$
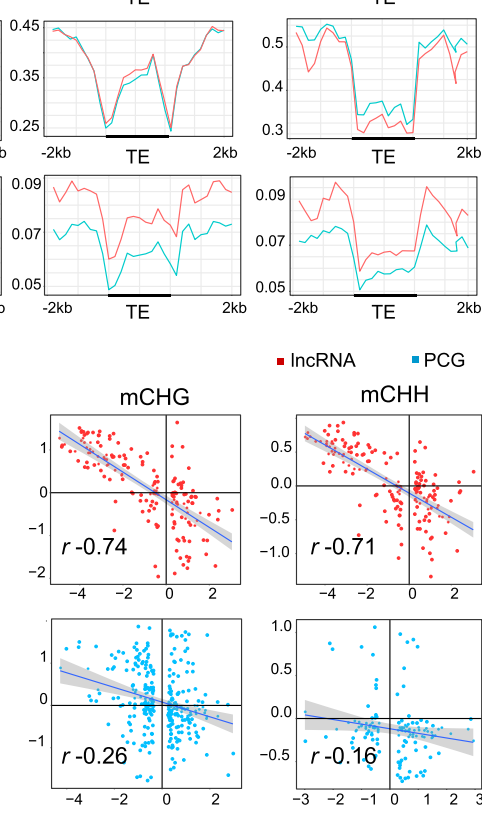

Log10(FPKM(Parent)/FPkM(F1))
B

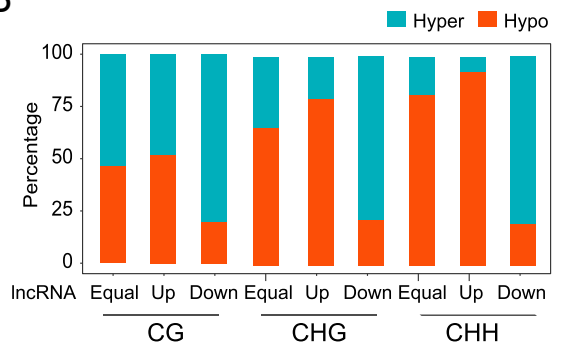

E
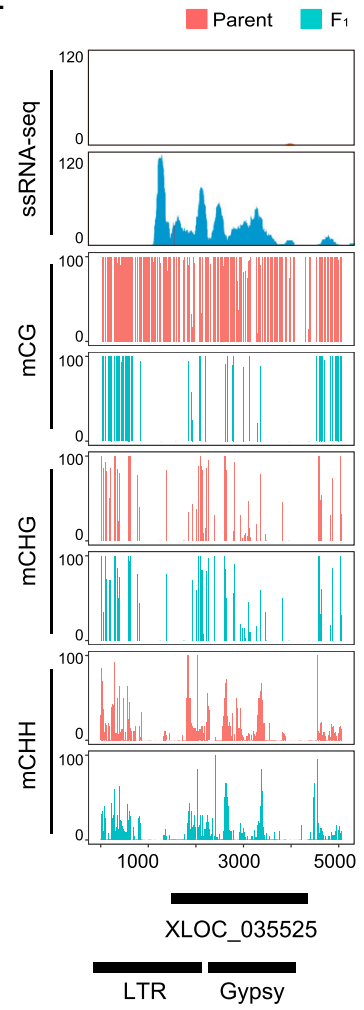

Fig. 6 DNA methylation level is negatively associated with IncRNA expression in $\mathrm{F}_{1}$ hybrid. a Pie chart showing the distribution of differently methylated regions (DMRs) of IncRNAs in categories of $\mathrm{CG}, \mathrm{CHG}$, and $\mathrm{CHH}$ in $\mathrm{F}_{1}$ hybrid. LncRNAs were classified into groups of equal expressed (Equal, $n=2610$ ), upregulated (Up, $n=1,302, p<0.01$, fold change $>2$ ), and downregulated (Down, $n=1,272, p<0.01$, fold change $<0.5$ ). $\mathbf{b}$ Histogram showing the proportion of hyper- and hypo-methylation on DMRs in IncRNAs. LncRNAs were classified into groups the same with a. c DNA methylation (CG, CHG) profile of equal expressed (Equal), upregulated (Up), and downregulated (Down) IncRNA in hybrid. Red and blue represent parent and $F_{1}$, respectively. $\mathbf{d}$ Correlation between differential gene expression $(p<0.01$ and fold change $>2.0)$ and differential DNA methylation $(p<0.01)$. e Example of $F_{1}$-upregulated IncRNA, XLOC_035525. Stack view of XLOC_035525 locus with RNA seq and DNA methylation in Ga and $F_{1}$

To confirm this observation, we examined the DMR patterns on differentially expressed lncRNAs by plotting DNA methylation distributions. As shown in Fig. 6c, the lncRNA-overlapped TEs were significantly less methylated in the upregulated lncRNAs in $\mathrm{F}_{1}$, which was true in all three DNA methylation contents (Fig. 6c). The $\mathrm{CHH}$ methylation on lnRNAs was constantly low in the $\mathrm{F}_{1}$, which was consistent with the hypo-methylation status in general. This trend suggested the RdDM might be active on lncRNA genes in $F_{1}$ hybrid.
DNA methylation is negatively associated with IncRNA expression in $F_{1}$ hybrid

Although genome-wide DMRs were identified in multiple hybridization tests, it is still inconclusive whether DNA methylation is associated with PCG expression changes in hybridization. We performed a correlation test for DNA methylation changes versus PCG and lncRNA expression changes in $F_{1}$. There was no correlation between DNA CG methylation and the expression of PCGs in $\mathrm{F}_{1}\left(r=0.03, p<8.53 \times 10^{-02}\right)$ (Fig. 6d), but 
lncRNA was negatively correlated with DNA CG methylation $\left(r=-0.63, p<2.2 \times 10^{-16}\right)$ (Fig. 6d). The correlation remained significant for $\mathrm{CHG}$ and $\mathrm{CHH}$ methylation (CHG $r=-0.74, p<2.20 \times 10^{-16}$; $\mathrm{CHH}$ for, $\left.r=-0.71, p<2.2 \times 10^{-16}\right)$. The representative example of XLOC_035525 activated in $F_{1}$ clearly showed the difference in DNA methylation between the parent $G a$ and $F_{1}$ (Fig. 6e). We therefore concluded that the DNA methylation level changes on lncRNA-overlapped TE regions were the major cause of IncRNA expression changes in $\mathrm{F}_{1}$. Specific demethylated TE regions contributed to the origin of novel lncRNA in the $F_{1}$ genome.

\section{TE-derived IncRNAs as a source of functional genes}

Since hybridization stimulated the transcription of non-coding regions of the genome, we ask whether these non-coding transcripts were simply noises arising from the chaos of genome shock or fixed in the tetraploid population as a source of functional genes. To address this question, we examined the lncRNA expression profile of allotetraploid cotton in wild species: 4 land races and 40 cultivars (Additional file 1: Table S7) [63]. The lncRNA homologs in the diploid parents and $F_{1}$ were employed as a control. By comparing their expression activity in the putative diploid ancestors, we found that 1493 out of the 2280 lncRNAs (syntenic either with Ga or Gr, RPKM >0.5) were specifically expressed in Gh (Fig. 7a). IncRNA expression was relatively stable in the allotetraploid, not only in the cultivars, but also in the wild cotton yucatanense and land races (Fig. 7b, Additional file 1: Table S8). But lncRNA expression varied drastically between the diploid and allotetraploid species (Fig. 7a, b, Additional file 1: Table S8). These results suggest that the genome shock of polyploidization introduced a significant variation in lncRNA expression similar to the effect of hybridization between the diploid species.

To further investigate whether the non-coding transcripts stimulated by genome shock have potential functions, we selected 10 lncRNAs (Additional file 1: Table S9) from the ST and SA groups in Gh vs $F_{1}$ for functional tests and comparison. One lncRNA among these candidates, XLOC_409583, was expressed from a demethylated TE locus. The primary sequence of XLOC_409583 was identified in both the $\mathrm{D}_{\mathrm{T}}$ and $\mathrm{Gr}$ (D) genomes, while the $\mathrm{A}_{\mathrm{T}}$ subgenome lacked an apparent orthologous sequence (Fig. 7c). In the $\mathrm{D}_{\mathrm{T}}$ subgenome, XLOC_409583 originated from a LINE locus (Fig. 7d). In contrast to $F_{1}$ and its diploid ancestor $\mathrm{Gr}$, the active expression of XLOC_409583 in the cultivated upland tetraploid cotton TM-1was associated with the demethylation of LINE. The active expression of XLOC_409583 was also detected in the wild upland cotton yucatanense, 4 land races (latifolium, punctatum, morrilli, palmeri), and
40 up-land cotton cultivars (Fig. 7d and Additional file 1: Table S8), indicating that XLOC_409583 transcription is stable after polyploidization.

To refine our understanding of the biological role of XLOC_409583, we performed virus-induced gene silencing (VIGS) tests in TM-1. The plants that underwent XLOC_409583 silencing showed increased height compared to the control group, indicating that the novel lncRNA XLOC_409583 played a role in plant development in the tetraploid cotton genome $(n=15$ in each treatment, with two repetitions) (Fig. 7f, g). Discovery of the activation of XLOC_409583 by demethylation provides insight into the role of DNA demethylation in the emergence of novel lncRNA in hybrids and polyploids. Functional analysis of these novel lncRNAs will further uncover their biological significance in hybrids and polyploids [64].

RNA polymerase II is essential for the transcription of TEoverlapped IncRNA

To determine whether the variation in lncRNA expression seen in hybridization and polyploidization is affected by RNA transcriptase, we examined the lncRNA profile in the natural population of cotton. LncRNAs with mapping reads in the mRNA-Seq profiles were identified as Poly (A)+. Eighty-five cotton mRNA-seq were scanned in total $[44,63]$. We found most IncRNA $(84.55 \%)$ could be detected in the Poly (A)+ library (Fig. 8a), which was in agreement with previous reports for rice and maize [63].

To confirm that IncRNAs were transcribed by RNA polymerase II (Pol II), we used Pole II antibody to pull down the binding DNA fragments in diploid cotton $(\mathrm{Ga}$ and $\mathrm{Gr}$ ), the $\mathrm{F}_{1}$, and the allotetraploid (Gh) cotton species (Additional file 1: Table S10). Then, using the model-based analysis for ChIP-Seq, we identified 19527576 high-confidence peaks (Additional file 1: Table S11). Pol II signals were enriched on both lncRNA and PCG in similar patterns (Fig. 8b, Additional file 2: Figure S8). Compared to the diploid parents, the binding efficiency of Pol II in $F_{1}$ is not associated with transcription efficiency on either lncRNA loci or on PCGs (Pearson's correlation test, $p>0.05$ ). In addition, most Pol II-associated lncRNA transcripts contain TEs (Fig. 8c). These observations suggest that Pol II is the major RNA polymerase binding to lncRNA loci, especially on TEs (Fig. 8d). These results imply that, in addition to Pol IV and V, Pol II is also involved with TE transcription.

\section{Discussion}

Interspecies hybrid is a model for the study of IncRNA evolution

Evolutionary conservation of lncRNA is poorly understood due to the lack of sufficiently close species with 

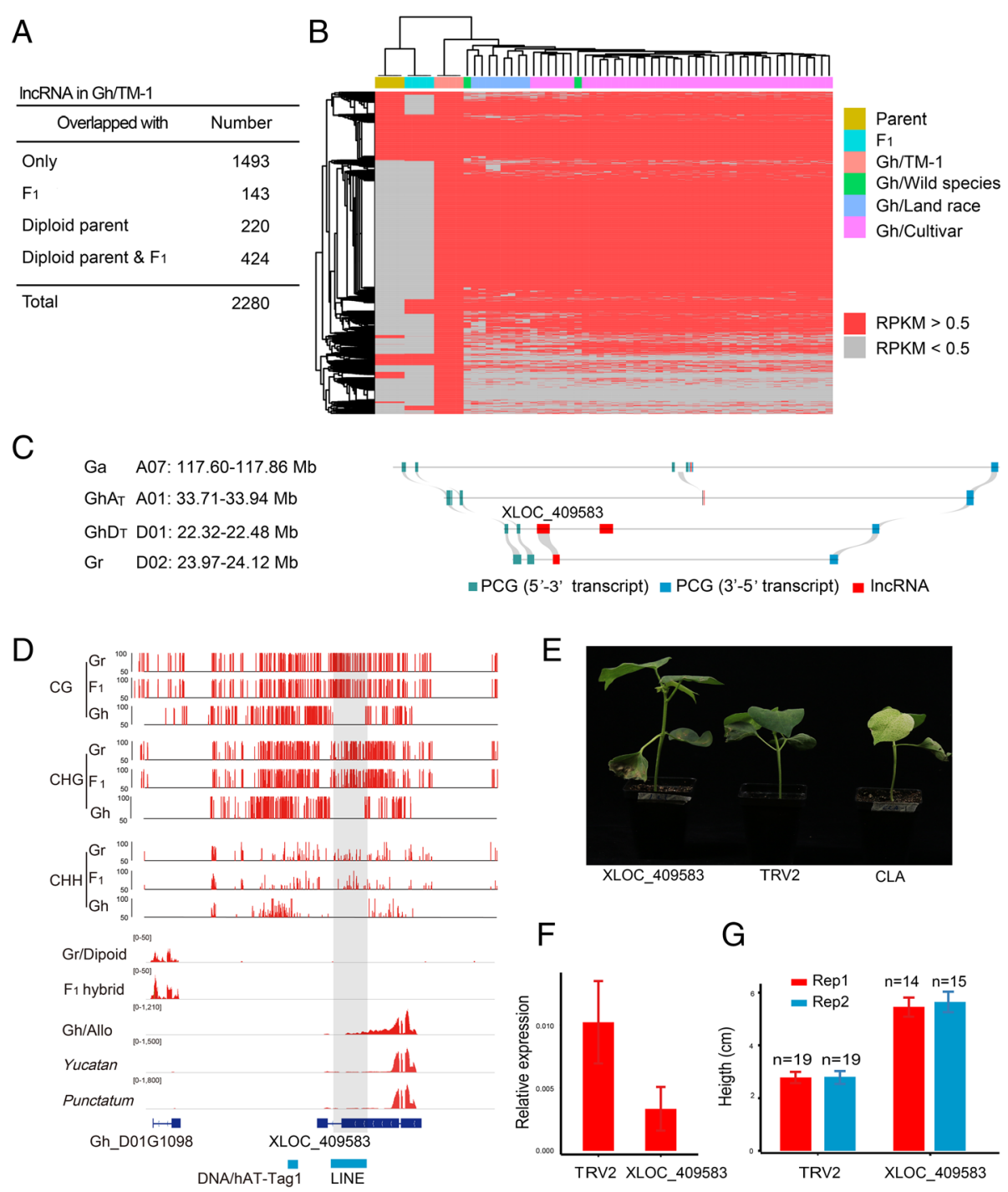

Fig. 7 Polyploidization-stimulated IncRNAs have potential function. a Table showing number of Gh IncRNA overlapped with parent and $F_{1}$. Total number of SA and ST IncRNA of Gh expressed in leaf (threshold RPKM > 0.5). b Heatmap showing IncRNA expressed in diploid parents, F1, Gh, upland cotton land races, and cultivars. c The syntenic relationship of XLOC_409583 in Gossypium spp. Gray curves connect the homologous regions between the corresponding chromosomes. The syntenic region was defined as the region with at least four aligned coding genes. XLOC_409583 is located on the $D_{T}$ subgenome (on chromosome of $\mathrm{GhD}_{T} \mathrm{D01}$ ), which is syntenic with the D diploid ancestor (on Gr Chr02). d Browser stacks for DNA methylation and RNA-seq of XLOC_409583 in Gr (diploid ancestor), F 1 hybrid, and TM-1 (allotetraploid). e Representative photo of TM-1 plants treated with virus-induced gene silencing (VIGS) constructs TRV2: XLOC409583, TRV2, and TRV2:CLrV. f Relative expression of XLOC409583 in VIGS-treated cotton group. Plants treated with an empty vector were used as a control group. $\mathbf{g}$ Histogram showing the height of XLOC_409583 in VIGS-treated cotton group. An empty vector was used as a control group. $n$ represents the number of plants in each block

finely sequenced genomes for study [33]. Taking advantage of the three published genomes of closely related cotton species [44-46], we identified that $83.97 \%$ of cotton lncRNAs were conserved in Gossypium spp. Furthermore, approximately 59.29 to $76.34 \%$ of PCGs were one-to-one syntenic in all four genomes. These highly syntenic genomes helped to identify the homologous lncRNA loci.

Using the collinear method, homologous lncRNA loci with low sequence similarities were identified at a high confidence level. Our research model applied to the $F_{1}$ and allotetraploid genomes was designed to facilitate the examination of homologous IncRNA loci. Therefore, the genome specificity observed in this system provided solid evidence of the fast turnover of lncRNA. We found that only $10.86-26.15 \%$ of syntenic lncRNA loci were constantly expressed in multiple cotton species. These results further confirm the previous report that in animal genomes both sequence divergence and expression turnover contribute to the species specificity of lncRNA [32]. 


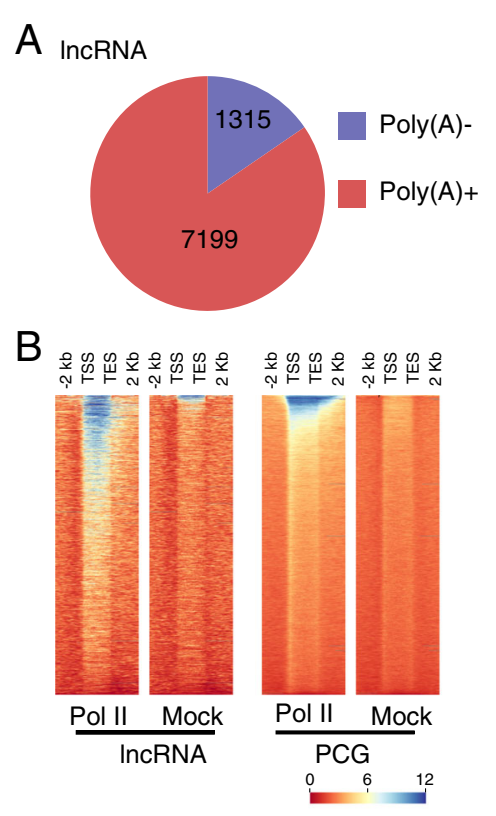

C

Equal $(n=227)$
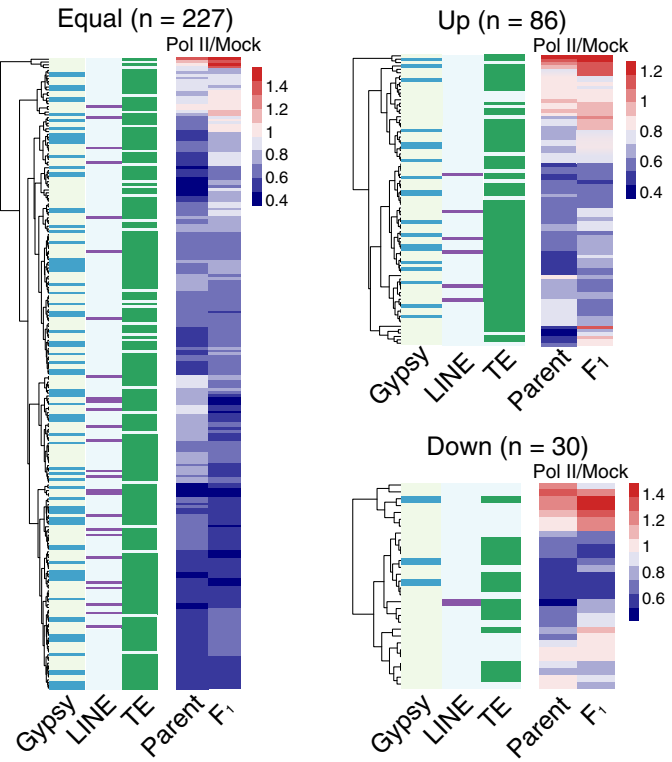

D
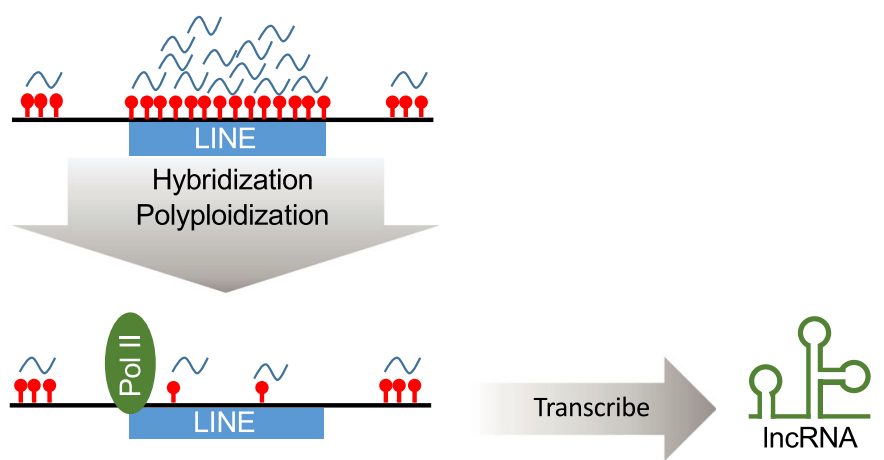

- gDNA

Transposon DNA methylation

$\sim$ SRNA

Fig. 8 Pol II is essential for transcription of TE-overlapped IncRNA. a Venn diagrams illustrating the proportion of IncRNAs with reads mapped against mRNA Seq library prepared with poly(A) enrichment (poly(A)+). Poly (A)- represents the unmapped IncRNAs. b Enrichment of Pol II signals at IncRNA and PCG sites. The heatmap reads are clustered according to the enrichment profile of Pol II and mock. c Heatmap showing the dynamics of Pol II binding efficiency difference in equal and up- and downregulated IncRNAs in the $F_{1}$ hybrid. The left panel of the heatmap indicates the annotation of IncRNA overlapped with Gypsy, LINE, and TE. $\mathbf{d}$ Schematic model of IncRNA origination within TE regions during genome shock of hybridization and polyploidization. $\mathbf{d}$ Transposon elements (TEs) are generally suppressed by siRNA and DNA methylation. During hybridization and polyploidization, de novo DNA methylation mediated by small RNA introduces dynamic RNA transcription. Based on our analysis, the reprogramming of DNA methylation during hybridization gives rise to novel IncRNAs from LINEs

\section{Rapid turnover of IncRNA in hybrid}

Hybridization and polyploidization are both common and crucial in genome evolution. Genome-wide changes can be ascribed to variations in PCG expression and alteration of epigenetic modifications, such as DNA methylation, histone modification, and sRNA generation. Most PCGs in synthetic allopolyploid are expressed at mid-parent level [5]. However, in this study, we found that IncRNA expression was changed dramatically in hybrid. Our data indicated that lncRNA was not substantially gained and lost during evolution, but was instead induced by the genomic shock of interspecies hybridization, provoking new species formation. Transcription of lncRNA underwent tremendous variation during genome shock. Given that lncRNAs participate in critical biological process, such as Xist silencing in animals [65] or miRNA target mimicry in plants [26], it is reasonable to assume that lncRNA reprograming in hybrids can affect genes regulated by non-coding RNAs. Therefore, we hypothesized that the rapid transcriptional turnover of lncRNAs might further affect the lineage-specific emergence or disappearance of specific traits. 
The epigenetic modifications on TE affect the IncRNA origin TEs have been reported to be involved in miRNA origin and evolution $[66,67]$. TEs also contribute to alternative gene structures such as novel promoters, splice sites, or polyadenylation signals [68]. Previous reports elucidated that TEs are major contributors to the origin of some lncRNA in vertebrates [38-40]. Many functional lncRNAs such as Xist [41], TUG1 [69], linc-ROR [70], PCAT-1 [24], and SLC7A2-IT1A [71] are overlapped with TEs. We also observed a strong correlation between TEs and lncRNAs along the evolutionary path from diploid to allotetraploid. In our simulation model of cotton evolution, lncRNAs tended to be retained with TEs, indicating the potential impact of TEs on IncRNA origin as well as heritability. We found that TEs exhibited biased distribution toward lncRNA loci rather than coding genes, and LINEs especially contributed disproportionally to lncRNAs in all cotton species.

As a mobile element, TEs are normally transcriptionally silent regions due to DNA methylation via RdDM. But TEs overlapping with lncRNA loci are transferred to a transcriptionally active status, implying a possible difference in local regulation or modification. The sRNA distribution pattern and DNA methylation levels of lncRNA loci in the $F_{1}$ hybrid confirmed that these regions were activated. Therefore, we hypothesized that the IncRNA loci originated from select TEs, such as LINEs, with few suppressive modifications. Based on our analysis, the de novo methylation as well as reprogramming of DNA methylation in hybridization created novel lncRNAs arising from LINEs (Fig. 8). A latest study on the epigenetic landscape of cancer cells finds that the lncRNA genes are hypo-methylated [25]. Some oncogenic lncRNA genes are under the diverse epigenetic modifications, such as CpG methylation. The de-methylated lcnRNA gene EPIC1 can promote the cell propagation in cancer [25]. These reports suggested that the DNA methylation-directed lncRNA regulation is a general mechanism both in plant and animal genomes.

\section{Materials and methods}

\section{Materials}

Interspecies hybrids of Gossypium arboreum (AA, $2 \mathrm{n}=$ $2 \mathrm{x}=26$ ) and $\mathrm{G}$. raimondii (DD, $2 \mathrm{n}=2 \mathrm{x}=26$ ) were generated by hand pollination. Three biological replicates of 0 DPA (days post anthesis) ovules and leaves from each of G. arboreum, G. raimondii, interspecies hybrid (G. arboreum $\times$ G. raimondii) $\mathrm{F}_{1}$, and $\mathrm{G}$. hirsutum (AADD, $2 n=4 x=52$ ) were collected from the greenhouse of Nanjing Agricultural University. All plants were under the same controlled growing conditions at $25^{\circ} \mathrm{C}, 16 / 8 \mathrm{~h}$ day/night. Samples were frozen in liquid nitrogen immediately upon collection and stored at $-70{ }^{\circ} \mathrm{C}$ in preparation for RNA isolation.

\section{LncRNA library construction and sequencing}

Total RNA was isolated from the plant tissues using the Spectrum Plant Total RNA Kit (Sigma-Aldrich). After RNA isolation, ribosomal RNA was removed using the Epicentre Ribo-zero ${ }^{\mathrm{TM}}$ rRNA Removal Kit (Epicentre, USA). Next, sequencing libraries were generated from rRNA-depleted RNA using the NEBNext ${ }^{\odot}$ Ultra $^{\mathrm{Tm}}$ Directional RNA Library Prep Kit for Illumina ${ }^{\oplus}$ (NEB, USA). Finally, strand-specific sequencing was performed with the Illumina HiSeq 2000 system (paired-end 125-bp reads).

\section{Pipeline for IncRNA identification}

All strand-specific RNA-seq reads were quality-trimmed and quality-filtered using fastx_toolkit (http://hannonl ab.cshl.edu/fastx_toolkit/). Clean reads were then mapped separately to the corresponding references using TopHat (v.2.0.14) $(N=2$, library type $=$ fr-first strand $)$. The genome sequences and annotation files for G. arboreum (v.2.0), G. raimondii, and G. hirsutum (v.1.1) were collected from the Cotton Genome Project (CGP: https://www.ncbi.nlm.nih. gov/genome/?term=Gossypium+arboreum), Phytozome (v.9 .0) (http://www.phytozome.net), and the website (http:// mascotton.njau.edu.cn/Data.htm), respectively. The $F_{1}$ genome was generated by mixing the genome sequences of $G$. arboretum and G. raimondii. The transcripts from each dataset were assembled independently using the Cufflinks program (v.2.2.1) $(-\mathrm{u}$, library type $=$ fr-first strand $)$ and Scripture program $(-$-coverage $=0.2)[53,72]$. All transcripts from each species were merged to generate final transcripts using Cuffmerge [53]. Transcripts less than 200 nt were discarded. Using Cuffcompare, transcripts were given the class code " $u$," " $x$," or " $i$," representing intergenic sequences, antisense sequences of known genes, and intronic sequences, respectively [53]. The Coding Potential Calculator (CPC) software was used to calculate the coding potential of the remaining transcripts [73]. All transcripts with CPC scores $>0$ were discarded. The remaining transcripts were subjected to HMMER (v. 3.1b2) in order to exclude transcripts containing known protein domains (cutoff <0.001) [74]. The remaining transcripts were candidate lncRNAs. To reduce the isoform complexity of lncRNA, only the longest transcript of each loci was used for further analysis.

\section{Identification of transposable element-derived IncRNA}

We annotated transposable elements in the genome using RepeatMasker (v.4.0.6) (http://www.repeatmasker.org). RepeatModeler (v.1.0.8) (http://www.repeatmasker.org/ RepeatModeler.html) was used to create three de novo transposable element (TE) libraries based on the G. raimondii, G. arboreum, and G. hirsutum reference genomes using default parameters. We then used RepeatMasker to identify repeat elements using both the de novo libraries and the MIPS repeat database (mipsREdat_9.3p) [75]. The annotation from RepeatMasker was then parsed to 
exclude low complexity and non-TE repeats. Next, transposons were classified into Gypsy, Copia, LTR, LINE, DNA, unknown, and other categories. LncRNA-derived TEs were identified by determining overlapping genomic coordinates of TEs or TE fragments of at least $1 \mathrm{bp}$ using the intersectBed program from BEDTools (v.2.17.0) [54]. When multiple TE features were found for a single IncRNA, the longer TE feature was counted.

\section{Identification of ST and SA IncRNAs}

ST lncRNA was reconstructed based on sequence similarity and position. NATs and intronic RNAs that overlapped protein-coding gene (PCG) loci were removed. Then the PCGs and lincRNAs in different subgenomes were reciprocally aligned using BLASTN (v.2.2.27) ( $E$ value $<1 \times 10^{-10}$, -max_target_seq 1) [76]. ST IncRNA, in syntenic blocks between two subgenomes, were identified using the MCScanX (-b 2, -s 5) [77]. To identify SA IncRNA, the IncRNA sequences of species A were aligned to the syntenic blocks of species B using BLASTN ( $E$ value $<1 \times 10^{-10}$, -max_target_seq 1) [76].

\section{Expression analysis}

HTSeq-count software (v.0.6.0) [78] was used to obtain read counts for each lncRNA or gene module (-s yes $-\mathrm{m}$ union). Read counts were normalized to RPKM (reads per kilobase per million reads). To assess the accumulated gene expression divergence between the parent lines and the hybrid $F_{1}$, an in silico parental mix was constructed by combining clean reads of G. raimondii and $G$.arboreum at a ratio of 1:1. Spearman's correlation between biological replicates was calculated using $R$ from the RPKM values. Differentially expressed transcripts were calculated using the R package, edgeR [79].

\section{Small RNA library construction and sequencing}

Total RNA was extracted from the 0 DPA ovules and leaves of two biological replicates. Small RNAs were then separated from total RNA by polyacrylamide gel electrophoresis. Three micrograms of total RNA per sample was used as the input material for construction of the small RNA library. Sequencing libraries were generated using NEBNext ${ }^{\circ}$ Multiplex Small RNA Library Prep Set for Illumina ${ }^{\circ}$ (NEB, USA), following the manufacturer's recommendations. The library preparations were sequenced on an Illumina HiSeq 2000 platform and 50-bp single-end reads were generated.

\section{Processing of sRNA sequencing data}

After sRNA sequencing, adapters and low-quality nucleotides were trimmed from the data. sRNA clean reads were then aligned with the $F_{1}$ genome (a mixture of the $\mathrm{Ga}$ and $\mathrm{Gr}$ genomes) using Bowtie, with no mismatch $(-\mathrm{m} 50,-\mathrm{v} 0)$ [80]. Any aligned small RNA reads that mapped to more than 50 loci were removed. The remaining mapped reads were aligned with noncoding RNAs using Rfam release (http://rfam.sanger.ac.uk/) and the known miRNA database in miRBase release 19 (http://www.mirbase.org/) [81], in order to identity miRNA, snRNA, tRNA, and rRNA. miREvo [82] and mirdeep2 software [83] were integrated to predict novel miRNAs. All reads originating from miRNA, TAS genes, rRNA, tRNA, snRNA, and snoRNA were removed. The remaining 20-25-nt-long reads were selected as siRNA. The distribution of siRNA across different features was drawn using deeptools [84].

\section{Analysis of MethylC-seq reads}

MethylC-seq and differentially methylated regions (DMRs) were retrieved from a previous study in the supplemental information (https://genomebiology.biomedcentral.com/ articles/10.1186/s13059-017-1229-8) [60]. The methylation level across different features was calculated using an in-house perl and $\mathrm{R}$ script.

\section{Virus-induced gene silencing technology}

A 300-bp fragment of XLOC409583 was amplified (F primer, AATAAG TGTGAAATTGTCGGGC; R primer, ATTCATGGCGATAAAGTCGGA) and cloned to form a $\mathrm{XbaI} / \mathrm{Bam} \mathrm{HI}$-digested pTRV2 vector, creating a VIGS vector named $p T R V 2-$ XLOC409583 (F primer: ATTC TGTGAGTAAGGTTACCGAATTCGAAA GTCCTTC GCTACAAAT; R primer: AGACGCGTGAGCTCGGTA CCGGATCC ACTATTGCCAATCGTCTTCA). The vectors $p T R V 1$ and $p T R V 2-X L O C 409583$ were then transformed by the Agrobacterium strain GV3101 via electroporation (Bio-Rad, Hercules, CA, USA) [85]. For the VIGS assay, the transformed Agrobacterium colonies were incubated overnight at $28{ }^{\circ} \mathrm{C}$ in an antibiotic selection medium containing $50 \mathrm{mg} / \mathrm{L}$ rifampicin and $50 \mathrm{mg} / \mathrm{L}$ kanamycin. Agrobacterium cells were centrifuged and resuspended in infiltration buffer $(10 \mathrm{mM} \mathrm{MgCl} 2,10 \mathrm{mM}$ MES, and $200 \mathrm{mM}$ acetosyringone), adjusted to an $\mathrm{OD}_{600}$ $=0.5$. Agrobacterium strains containing $p T R V 1$ and $p T R V 2$ vectors were mixed in a ratio of 1:1. Seedlings with mature cotyledons but without a visible true leaf ( 7 days after germination) were infiltrated by inserting the Agrobacterium suspension into the cotyledons via syringe. The plants were grown in pots at $25{ }^{\circ} \mathrm{C}$ in a growth chamber under a $16 / 8 \mathrm{~h}$ light/dark cycle with $60 \%$ humidity. For each treatment group, 32 individual plants were employed.

\section{RNA extraction and qRT-PCR}

RNA was extracted from leaf tissue and treated with a BioFlux kit. First-strand cDNA was generated using TransScript One-Step gDNA Removal and cDNA Synthesis SuperMix (TransGen Biotec Co., Ltd.), according to the manufacturer's instructions. Quantitative RT-PCRs were 
performed with the primers F: CCTTGTCAGAGTCC TCTGGTAG; R: GAGTTGAATGGGCATTCTTG.

\section{Chromatin immunoprecipitation and sequencing (ChIP-Seq)} Chromatin immunoprecipitation (ChIP) was performed as described and with several adaptations [86]. One gram of leaves for each sample of $\mathrm{AA}, \mathrm{DD}, \mathrm{AD}$, and $\mathrm{AADD}$ genomes was used in the assay. After plant material crosslinking, nuclei isolation, cell lysis, and chromatin sonication as described in the protocol, ChIP reaction was performed using Anti-RNA polymerase II antibody (ABcam, Anti-RNA polymerase II CTD repeat YSPTSPS antibody [8WG16] - ChIP Grade, ab817) and protein A+G magnetic beads (Millpore), referred to as the "ChIP" group. The control group for each sample was set up similarly with the experimental group using sonicated chromatin with protein $A+G$ magnetic beads but without antibody, referred to as the "Mock" group, which served as the background of the ChIP reaction. The ChIP reaction was performed overnight at $4^{\circ}$ with gentle rotation, followed by separation and washing of beads using magnetic separation device (Millpore Magna GrIP Rack). DNA purification was performed using a commercial spin column kit. To verify the DNA enrichment, ChIP-Seq libraries were constructed with the NEBNext ChIP-Seq Library Prep Master Mix Set for Illumina (NEB) using NEBNext Multiplex Oligos for Illumina (NEB). DNA libraries including the "ChIP" and "Mock" groups respectively for each sample were pair-end sequenced with 150 bp reads using an Illumina HiSeq2500.

\section{Analysis of ChIP-Seq}

All ChIP-Seq reads were quality-trimmed and quality-filtered using fastx_toolkit (http://hannonlab.csh .edu/fastx_toolkit/). Clean reads were then mapped separately to the corresponding references using Bowtie (v.2.0.14) with no mismatch [80]. Peak calling analysis was used for model-based analysis for ChIP-seq (MACS) [87]. Profile of ChIP-Seq in PCG and lncRNA was visualized using Deeptools with default parameter [84].

\section{Scan IncRNA expression variation in upland cotton population}

One wild upland cotton (accession yucatanense), 4 land races (latifolium, punctatum, morrilli, palmeri) and 40 up-land cotton cultivars were download from SRA project SRX2326742 [63] (Additional file 1: Table S7). Reads were quality-trimmed and quality-filtered using fastx_toolkit (http://hannonlab.cshl.edu/fastx_toolkit/). Clean reads were then mapped to the corresponding references of Gossypium hirsutum using TopHat (v.2.0.14) [53]. HTSeq-count software (v.0.6.0) [78] was used to obtain read counts for each lncRNA or gene module $(-\mathrm{s}$ yes $-\mathrm{m}$ union), then read counts were normalized to RPKM.
Expressed lncRNA were determined by applying a threshold of RPKM > 0.5.

\section{Additional files}

Additional file 1: Table S1. The strand-specific RNA sequencing (ssRNAseq) library data summary. Table S2. The total IncRNA predicted in Gossypium species. Table S3. The total IncRNA predicted in Gossypium species. Table S4. Summary of the syntenic blocks of Gossypium spp. Table S5. The small RNA sequencing library data summary. Table S6. Classification of sRNA. Table S7. Eighty-five Poly(A)+ RNA-seq library of Gossypium hirsutum. Table S8. FPKM of Gh syntenic IncRNA. Table S9. Candidate IncRNAs. Table S10. Summary of ChIP-Seq library. (XLSX $1135 \mathrm{~kb}$ )

Additional file 2: Figure S1. LncRNA landscape in Gossypium spp., $G$. arboreum, G. raimondii, (G. arboreum $\times$ G. raimondii) F1, and G. hirsutum. Figure S2. Sequence similarity of IncRNAs across different species. Figure S3. Low similarity of syntenic and transcribed IncRNA. Figure S4. Expression correlation coefficient of different TE-overlapped IncRNA. Figure S5. LncRNA were transcribed from siRNA-depleted LINEs/TEs. Figure S6. Profile of 21 and 24 nt leaf sRNA. Figure S7. Profile of 21 and 24 nt ovule sRNA. Figure S8. Heatmaps showing the dynamics of Pol II binding signals in IncRNA and PCG features across the four cotton species. (PDF $2882 \mathrm{~kb}$ )

\section{Acknowledgements}

We thank CAAS Cotton Research Institute for providing the G. raimondii cotton ovule and leaf tissue used in the present study. We thank Prof. Xiaoya Chen and Yingbo Mao from SIPPE for kindly sharing the data of G. hirsutum Zhong12 and Zhong12 GL. We thank Dr. Qingxin Song for the insightful suggestions and kindly sharing the DNA methylation analysis data. We thank Pengchuan Sun from North China University of Science and Technology for generous advices on data visualization.

\section{Funding}

This work was financially supported in part by grants from the National Key Research and Development Program (2016YFD0101006), the National Natural Science Foundation of China (NSFC, 31600989), the Ministry of Science and Technology (2016YFA0500800), the Natural Science Foundation of Jiangsu Province (BK20150653), and the JCIC-MCP project.

\section{Availability of data and materials}

The IncRNA sequences and genome coordinate files can be accessed from github repositories (https://github.com/epi-cotton/LncRNA-in-polyploid-cotton) [88]. Sequences of ssRNA-seq and Chip-seq have been deposited in the NCBI Nucleotide and Sequence Read Archive (SRA) under the accession PRJNA373801 [89]. Sequences of sRNA-seq have been deposited in the Sequence Read Archive (SRA) under the accession PRJNA375828 [90]. BS-seq are publicly available under the accession SRP071640 [59]. For the comparison with rRNA-depleted RNA-seq, 35 tissues of Gossypium hirsutum were downloaded from SRA project SRP044705 [44]. To scan IncRNA expression variation in upland cotton population, one wild upland cotton (accession yucatanense), 4 land races (latifolium, punctatum, morrilli, palmeri), and 40 upland cotton cultivars were download from SRA project SRX2326742 [62]. DMRs of BS-seq were downloaded from the link (https://genomebiology.biomedcen tral.com/articles/10.1186/s13059-017-1229-8).

\section{Authors' contributions}

$X G$ and BZ conceptualized the research program. XG designed experiments and coordinated the project. TZ and SF collected the tissues. XT conducted the ChIP-Seq operation. TZ and XG analyzed all data and wrote the manuscript. $\mathrm{HH}$ prepared the Zhong 12 and Zhong12 GL material and ssRNA seq data. BZ provided the $\left(G\right.$. arboreum $\times G$. raimondii) $F_{1}$ leaf and ovule tissue for the study. $\mathrm{TZ}, \mathrm{XG}$, and LW visualized the data. WM, GS, SG, and YH conducted the experiments. All authors discussed the results and commented on the manuscript. All authors read and approved the final manuscript. 


\section{Competing interests}

The authors declare that they have no competing interests.

\section{Publisher's Note}

Springer Nature remains neutral with regard to jurisdictional claims in published maps and institutional affiliations.

\section{Author details}

${ }^{1}$ State Key Laboratory of Crop Genetics and Germplasm Enhancement, Cotton Hybrid R \& D Engineering Center (the Ministry of Education), College of Agriculture, Nanjing Agricultural University, Nanjing 210095, Jiangsu, China. ${ }^{2}$ College of Agriculture and Biotechnology, Zhejiang University, Zhejiang 210058, Hangzhou, China. ${ }^{3}$ National Key Laboratory of Plant Molecular Genetics, National Plant Gene Research Center, Institute of Plant Physiology and Ecology, Shanghai Institutes for Biological Sciences, Chinese Academy of Sciences, Shanghai 200032, China.

Received: 26 July 2018 Accepted: 23 October 2018 Published online: 12 November 2018

\section{References}

1. Jiao Y, Wickett NJ, Ayyampalayam S, Chanderbali AS, Landherr L, Ralph PE, Tomsho LP, Hu Y, Liang H, Soltis PS, et al. Ancestral polyploidy in seed plants and angiosperms. Nature. 2011;473:97-100.

2. Chen ZJ. Genomic and epigenetic insights into the molecular bases of heterosis. Nat Rev Genet. 2013;14:471-82.

3. Song Q, Chen ZJ. Epigenetic and developmental regulation in plant polyploids. Curr Opin Plant Biol. 2015;24:101-9.

4. Li A, Liu D, Wu J, Zhao X, Hao M, Geng S, Yan J, Jiang X, Zhang L, Wu J, et al. mRNA and small RNA transcriptomes reveal insights into dynamic homoeolog regulation of allopolyploid heterosis in nascent hexaploid wheat. Plant Cell. 2014;26:1878-900.

5. Yoo MJ, Szadkowski E, Wendel JF. Homoeolog expression bias and expression level dominance in allopolyploid cotton. Heredity (Edinb). 2013;110:171-80.

6. Tran RK, Zilberman D, de Bustos C, Ditt RF, Henikoff JG, Lindroth AM, Delrow J, Boyle T, Kwong S, Bryson TD, et al. Chromatin and siRNA pathways cooperate to maintain DNA methylation of small transposable elements in Arabidopsis. Genome Biol. 2005;6:R90.

7. Zheng X, Zhu J, Kapoor A, Zhu JK. Role of Arabidopsis AGO6 in siRNA accumulation, DNA methylation and transcriptional gene silencing. EMBO J. 2007;26:1691-701.

8. Havecker ER, Wallbridge LM, Hardcastle TJ, Bush MS, Kelly KA, Dunn RM, Schwach F, Doonan JH, Baulcombe DC. The Arabidopsis RNA-directed DNA methylation argonautes functionally diverge based on their expression and interaction with target loci. Plant Cell. 2010;22:321-34.

9. Ha M, Lu J, Tian L, Ramachandran V, Kasschau KD, Chapman EJ, Carrington JC, Chen X, Wang XJ, Chen ZJ. Small RNAs serve as a genetic buffer against genomic shock in Arabidopsis interspecific hybrids and allopolyploids. Proc Natl Acad Sci U S A. 2009;106:17835-40.

10. Barber WT, Zhang W, Win H, Varala KK, Dorweiler JE, Hudson ME, Moose SP. Repeat associated small RNAs vary among parents and following hybridization in maize. Proc Natl Acad Sci U S A. 2012;109:10444-9.

11. Groszmann M, Greaves IK, Albertyn ZI, Scofield GN, Peacock WJ, Dennis ES. Changes in 24-nt siRNA levels in Arabidopsis hybrids suggest an epigenetic contribution to hybrid vigor. Proc Natl Acad Sci U S A. 2011;108:2617-22.

12. Madlung A, Masuelli RW, Watson B, Reynolds SH, Davison J, Comai L. Remodeling of DNA methylation and phenotypic and transcriptional changes in synthetic Arabidopsis allotetraploids. Plant Physiol. 2002;129:733-46.

13. He G, Chen B, Wang X, Li X, Li J, He H, Yang M, Lu L, Qi Y, Wang X, Deng $X W$. Conservation and divergence of transcriptomic and epigenomic variation in maize hybrids. Genome Biol. 2013;14:R57.

14. Chodavarapu RK, Feng S, Ding B, Simon SA, Lopez D, Jia Y, Wang GL, Meyers BC, Jacobsen SE, Pellegrini M. Transcriptome and methylome interactions in rice hybrids. Proc Natl Acad Sci U S A. 2012;109:12040-5.

15. Rigal M, Becker C, Pelissier T, Pogorelcnik R, Devos J, Ikeda Y, Weigel D, Mathieu $O$. Epigenome confrontation triggers immediate reprogramming of DNA methylation and transposon silencing in Arabidopsis thaliana F1 epihybrids. Proc Natl Acad Sci U S A. 2016;113:E2083-92.

16. Ng DW, Miller M, Yu HH, Huang TY, Kim ED, Lu J, Xie Q, McClung CR, Chen ZJ. A role for $\mathrm{CHH}$ methylation in the parent-of-origin effect on altered circadian rhythms and biomass heterosis in Arabidopsis intraspecific hybrids. Plant Cell. 2014;26:2430-40.

17. Ponting CP, Oliver PL, Reik W. Evolution and functions of long noncoding RNAs. Cell. 2009;136:629-41.

18. Iyer MK, Niknafs YS, Malik R, Singhal U, Sahu A, Hosono Y, Barrette TR, Prensner JR, Evans JR, Zhao S, et al. The landscape of long noncoding RNAs in the human transcriptome. Nat Genet. 2015;47:199-208.

19. Pauli A, Valen E, Lin MF, Garber M, Vastenhouw NL, Levin JZ, Fan L, Sandelin A, Rinn JL, Regev A, Schier AF. Systematic identification of long noncoding RNAs expressed during zebrafish embryogenesis. Genome Res. 2012;22:577-91.

20. Zhang YC, Liao JY, Li ZY, Yu Y, Zhang JP, Li QF, Qu LH, Shu WS, Chen YQ. Genome-wide screening and functional analysis identify a large number of long noncoding RNAs involved in the sexual reproduction of rice. Genome Biol. 2014;15:512.

21. Li L, Eichten SR, Shimizu R, Petsch K, Yeh CT, Wu W, Chettoor AM, Givan SA, Cole RA, Fowler JE, et al. Genome-wide discovery and characterization of maize long non-coding RNAs. Genome Biol. 2014;15:R40.

22. Liu J, Jung C, Xu J, Wang H, Deng S, Bernad L, Arenas-Huertero C, Chua NH. Genome-wide analysis uncovers regulation of long intergenic noncoding RNAs in Arabidopsis. Plant Cell. 2012;24:4333-45.

23. Duret L, Chureau C, Samain S, Weissenbach J, Avner P. The Xist RNA gene evolved in eutherians by pseudogenization of a protein-coding gene. Science. 2006;312:1653-5.

24. Prensner JR, lyer MK, Balbin OA, Dhanasekaran SM, Cao Q, Brenner JC, Laxman B, Asangani IA, Grasso CS, Kominsky HD, et al. Transcriptome sequencing across a prostate cancer cohort identifies PCAT-1, an unannotated lincRNA implicated in disease progression. Nat Biotechnol. 2011;29:742-9.

25. Wang ZH, Yang B, Zhang M, Guo WW, Wu ZY, Wang Y, Jia L, Li S, Xie W, Yang D, Network CGAR. IncRNA epigenetic landscape analysis identifies EPIC1 as an oncogenic IncRNA that interacts with MYC and promotes cellcycle progression in cancer. Cancer Cell. 2018;33:706-720.e9.

26. Franco-Zorrilla JM, Valli A, Todesco M, Mateos I, Puga MI, Rubio-Somoza I, Leyva A, Weigel D, Garcia JA, Paz-Ares J. Target mimicry provides a new mechanism for regulation of microRNA activity. Nat Genet. 2007:39:1033-7.

27. Wang Y, Fan X, Lin F, He G, Terzaghi W, Zhu D, Deng XW. Arabidopsis noncoding RNA mediates control of photomorphogenesis by red light. Proc Natl Acad Sci U S A. 2014;111:10359-64.

28. Ding J, Lu Q, Ouyang Y, Mao H, Zhang P, Yao J, Xu C, Li X, Xiao J, Zhang Q. A long noncoding RNA regulates photoperiod-sensitive male sterility, an essential component of hybrid rice. Proc Natl Acad Sci U S A. 2012;109:2654-9.

29. Nadal-Ribelles M, Sole C, Xu Z, Steinmetz LM, de Nadal E, Posas F. Control of Cdc28 CDK1 by a stress-induced IncRNA. Mol Cell. 2014;53:549-61.

30. Mach J. The long-noncoding RNA ELENA1 functions in plant immunity. Plant Cell. 2017;29:916.

31. Yatusevich R, Fedak H, Ciesielski A, Krzyczmonik K, Kulik A, Dobrowolska G, Swiezewski S. Antisense transcription represses Arabidopsis seed dormancy QTL DOG1 to regulate drought tolerance. EMBO Rep. 2017;18:2186-96.

32. Kutter C, Watt S, Stefflova K, Wilson MD, Goncalves A, Ponting CP, Odom DT, Marques AC. Rapid turnover of long noncoding RNAs and the evolution of gene expression. PLoS Genet. 2012;8:e1002841.

33. Ulitsky I. Evolution to the rescue: using comparative genomics to understand long non-coding RNAs. Nat Rev Genet. 2016;17:601-14.

34. Washietl S, Kellis M, Garber M. Evolutionary dynamics and tissue specificity of human long noncoding RNAs in six mammals. Genome Res. 2014;24:616-28.

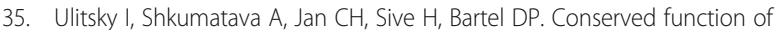
lincRNAs in vertebrate embryonic development despite rapid sequence evolution. Cell. 2011:147:1537-50.

36. Hezroni H, Koppstein D, Schwartz MG, Avrutin A, Bartel DP, Ulitsky I. Principles of long noncoding RNA evolution derived from direct comparison of transcriptomes in 17 species. Cell Rep. 2015;11:1110-22.

37. Wang X, Ai G, Zhang C, Cui L, Wang J, Li H, Zhang J, Ye Z. Expression and diversification analysis reveals transposable elements play important roles in the origin of Lycopersicon-specific IncRNAs in tomato. New Phytol. 2016;209:1442-55.

38. Davis MP, Carrieri C, Saini HK, van Dongen S, Leonardi T, Bussotti G, Monahan JM, Auchynnikava T, Bitetti A, Rappsilber J, et al. Transposondriven transcription is a conserved feature of vertebrate spermatogenesis and transcript evolution. EMBO Rep. 2017;18:1231-47.

39. Kelley D, Rinn J. Transposable elements reveal a stem cell-specific class of long noncoding RNAs. Genome Biol. 2012;13:R107. 
40. Kapusta A, Kronenberg Z, Lynch VJ, Zhuo X, Ramsay L, Bourque G, Yandell $M$, Feschotte $C$. Transposable elements are major contributors to the origin, diversification, and regulation of vertebrate long noncoding RNAs. PLOS Genet. 2013;9:e1003470.

41. Elisaphenko EA, Kolesnikov NN, Shevchenko Al, Rogozin IB, Nesterova TB, Brockdorff N, Zakian SM. A dual origin of the Xist gene from a proteincoding gene and a set of transposable elements. PLoS One. 2008:3:e2521.

42. Wan Q, Guan X, Yang N, Wu H, Pan M, Liu B, Fang L, Yang S, Hu Y, Ye W, et al. Small interfering RNAs from bidirectional transcripts of GhMML3_A12 regulate cotton fiber development. New Phytol. 2016;210:1298-310.

43. Schnable PS, Ware D, Fulton RS, Stein JC, Wei F, Pasternak S, Liang C, Zhang J, Fulton L, Graves TA, et al. The B73 maize genome: complexity, diversity, and dynamics. Science. 2009;326:1112-5.

44. Zhang T, Hu Y, Jiang W, Fang L, Guan X, Chen J, Zhang J, Saski CA, Scheffler BE, Stelly DM, et al. Sequencing of allotetraploid cotton (Gossypium hirsutum L. acc. TM-1) provides a resource for fiber improvement. Nat Biotechnol. 2015:33:531-7. https://doi.org/10.1038/nbt.3207.

45. Paterson AH, Wendel JF, Gundlach H, Guo H, Jenkins J, Jin D, Llewellyn D, Showmaker KC, Shu S, Udall J, et al. Repeated polyploidization of Gossypium genomes and the evolution of spinnable cotton fibres. Nature. 2012;492:423-7.

46. Li F, Fan G, Wang K, Sun F, Yuan Y, Song G, Li Q, Ma Z, Lu C, Zou C, et al. Genome sequence of the cultivated cotton Gossypium arboreum. Nat Genet. 2014;46:567-72.

47. Wicker T, Sabot F, Hua-Van A, Bennetzen JL, Capy P, Chalhoub B, Flavell A, Leroy $\mathrm{P}$, Morgante $\mathrm{M}$, Panaud $\mathrm{O}$, et al. A unified classification system for eukaryotic transposable elements. Nat Rev Genet. 2007:8:973-82.

48. Wendel JF, Cronn RC. Polyploidy and the evolutionary history of cotton. Adv Agron. 2003;78:139-86.

49. Zhao T, Wang L, Li S, Xu M, Guan X, Zhou B. Characterization of conserved circular RNA in polyploid Gossypium species and their ancestors. FEBS Lett. 2017;591:3660-9.

50. Wang M, Yuan D, Tu L, Gao W, He Y, Hu H, Wang P, Liu N, Lindsey K, Zhang $X$. Long noncoding RNAs and their proposed functions in fibre development of cotton (Gossypium spp.). New Phytol. 2015;207:1181-97.

51. Kumar S, Stecher G, Suleski M, Hedges SB. TimeTree: a resource for timelines, Timetrees, and divergence times. Mol Biol Evol. 2017;34:1812-9.

52. Yoo MJ, Wendel JF. Comparative evolutionary and developmental dynamics of the cotton (Gossypium hirsutum) fiber transcriptome. PLoS Genet. 2014; 10:e1004073.

53. Trapnell C, Roberts A, Goff L, Pertea G, Kim D, Kelley DR, Pimentel H, Salzberg SL, Rinn JL, Pachter L. Differential gene and transcript expression analysis of RNA-seq experiments with TopHat and Cufflinks. Nat Protoc. 2012;7:562-78.

54. Quinlan AR, Hall IM. BEDTools: a flexible suite of utilities for comparing genomic features. Bioinformatics. 2010;26:841-2.

55. Martienssen RA, Colot V. DNA methylation and epigenetic inheritance in plants and filamentous fungi. Science. 2001;293:1070-4.

56. Bird A. DNA methylation patterns and epigenetic memory. Genes Dev. 2002;16:6-21.

57. Chan SW, Henderson IR, Jacobsen SE. Gardening the genome: DNA methylation in Arabidopsis thaliana. Nat Rev Genet. 2005;6:351-60.

58. Law JA, Jacobsen SE. Establishing, maintaining and modifying DNA methylation patterns in plants and animals. Nat Rev Genet. 2010;11:204-20.

59. Lu J, Zhang C, Baulcombe DC, Chen ZJ: Maternal siRNAs as regulators of parental genome imbalance and gene expression in endosperm of Arabidopsis seeds. Proc Natl Acad Sci U S A. 2012;109:5529-34.

60. Song Q, Zhang T, Stelly DM, Chen ZJ. Epigenomic and functional analyses reveal roles of epialleles in the loss of photoperiod sensitivity during domestication of allotetraploid cottons. Genome Biol. 2017;18:99. https:// doi.org/10.1186/s13059-017-1229-8; DMRs between F1 hybrid and the parents (Gr/Ga). https://genomebiology.biomedcentral.com/articles/10.1186/ s13059-017-1229-8.

61. Kim KD, El Baidouri M, Abernathy B, Iwata-Otsubo A, Chavarro C, Gonzales M, Libault M, Grimwood J, Jackson SA. A comparative epigenomic analysis of polyploidy-derived genes in soybean and common bean. Plant Physiol. 2015;168:1433-47.

62. Shen H, He H, Li J, Chen W, Wang X, Guo L, Peng Z, He G, Zhong S, Qi Y, et al. Genome-wide analysis of DNA methylation and gene expression changes in two Arabidopsis ecotypes and their reciprocal hybrids. Plant Cell. 2012;24:875-92.

63. Wang M, Tu L, Lin M, Lin Z, Wang P, Yang Q, Ye Z, Shen C, Li J, Zhang L, et al. Asymmetric subgenome selection and cis-regulatory divergence during cotton domestication. Nat Genet. 2017:49:579-87. https://doi.org/10.1038/ng.3807.
64. Wang H, Niu QW, Wu HW, Liu J, Ye J, Yu N, Chua NH. Analysis of noncoding transcriptome in rice and maize uncovers roles of conserved IncRNAs associated with agriculture traits. Plant J. 2015;84:404-16.

65. Brown CJ, Hendrich BD, Rupert JL, Lafreniere RG, Xing Y, Lawrence J, Willard HF. The human XIST gene - analysis of a $17 \mathrm{~kb}$ inactive X-specific RNA that contains conserved repeats and is highly localized within the nucleus. Cell. 1992;71:527-42

66. Li Y, Li C, Xia J, Jin Y. Domestication of transposable elements into MicroRNA genes in plants. PLoS One. 2011;6:e19212.

67. Zhou ZK, Wang Z, Li WY, Fang C, Shen YT, Li CC, Wu YS, Tian ZX. Comprehensive analyses of microRNA gene evolution in paleopolyploid soybean genome. Plant J. 2013;76:332-44.

68. Cowley M, Oakey RJ. Transposable elements re-wire and fine-tune the transcriptome. PLoS Genet. 2013;9:e1003234

69. Yang L, Lin C, Liu W, Zhang J, Ohgi KA, Grinstein JD, Dorrestein PC, Rosenfeld MG. ncRNA- and PC2 methylation-dependent gene relocation between nuclear structures mediates gene activation programs. Cell. 2011;147:773-88.

70. Loewer S, Cabili MN, Guttman M, Loh YH, Thomas K, Park IH, Garber M, Curran M, Onder T, Agarwal S, et al. Large intergenic non-coding RNA-RoR modulates reprogramming of human induced pluripotent stem cells. Nat Genet. 2010:42:1113-7.

71. Cartault F, Munier P, Benko E, Desguerre I, Hanein S, Boddaert N, Bandiera S, Vellayoudom J, Krejbich-Trotot $\mathrm{P}$, Bintner $\mathrm{M}$, et al. Mutation in a primateconserved retrotransposon reveals a noncoding RNA as a mediator of infantile encephalopathy. Proc Natl Acad Sci U S A. 2012;109:4980-5.

72. Guttman M, Garber M, Levin JZ, Donaghey J, Robinson J, Adiconis X, Fan L, Koziol MJ, Gnirke A, Nusbaum C, et al. Ab initio reconstruction of cell typespecific transcriptomes in mouse reveals the conserved multi-exonic structure of lincRNAs. Nat Biotechnol. 2010;28:503-10.

73. Kong L, Zhang Y, Ye ZQ, Liu XQ, Zhao SQ, Wei L, Gao G. CPC: assess the protein-coding potential of transcripts using sequence features and support vector machine. Nucleic Acids Res. 2007;35:W345-9.

74. Finn RD, Clements J, Eddy SR. HMMER web server: interactive sequence similarity searching. Nucleic Acids Res. 2011;39:W29-37.

75. Nussbaumer T, Martis MM, Roessner SK, Pfeifer M, Bader KC, Sharma S, Gundlach H, Spannagl M. MIPS PlantsDB: a database framework for comparative plant genome research. Nucleic Acids Res. 2013;41:D1144-51.

76. Altschul SF, Madden TL, Schaffer AA, Zhang J, Zhang Z, Miller W, Lipman DJ. Gapped BLAST and PSI-BLAST: a new generation of protein database search programs. Nucleic Acids Res. 1997;25:3389-402.

77. Wang Y, Tang H, Debarry JD, Tan X, Li J, Wang X, Lee TH, Jin H, Marler B, Guo H, et al. MCScanX: a toolkit for detection and evolutionary analysis of gene synteny and collinearity. Nucleic Acids Res. 2012;40:e49.

78. Anders S, Pyl PT, Huber W. HTSeq-a Python framework to work with highthroughput sequencing data. Bioinformatics. 2015;31:166-9.

79. Robinson MD, McCarthy DJ, Smyth GK. edgeR: a Bioconductor package for differential expression analysis of digital gene expression data. Bioinformatics. 2010;26:139-40.

80. Langmead B, Trapnell C, Pop M, Salzberg SL. Ultrafast and memory-efficient alignment of short DNA sequences to the human genome. Genome Biol. 2009;10:R25.

81. Griffiths-Jones S, Grocock RJ, van Dongen S, Bateman A, Enright AJ. miRBase: microRNA sequences, targets and gene nomenclature. Nucleic Acids Res. 2006;34:D140-4.

82. Wen M, Shen $Y$, Shi S, Tang T. miREvo: an integrative microRNA evolutionary analysis platform for next-generation sequencing experiments. BMC Bioinformatics. 2012;13:140.

83. Friedländer MR, Mackowiak SD, Li N, Chen W, Rajewsky N. miRDeep2 accurately identifies known and hundreds of novel microRNA genes in seven animal clades. Nucleic Acids Res. 2012:40:37-52.

84. Ramirez F, Dundar F, Diehl S, Gruning BA, Manke T. deepTools: a flexible platform for exploring deep-sequencing data. Nucleic Acids Res. 2014;42:W187-91.

85. Gao X, Wheeler T, Li Z, Kenerley CM, He P, Shan L. Silencing GhNDR1 and GhMKK2 compromises cotton resistance to Verticillium wilt. Plant J. 2011;66: 293-305.

86. Haring M, Offermann S, Danker T, Horst I, Peterhansel C, Stam M. Chromatin immunoprecipitation: optimization, quantitative analysis and data normalization. Plant Methods. 2007:3:11.

87. Zhang Y, Liu T, Meyer CA, Eeckhoute J, Johnson DS, Bernstein BE, Nusbaum C, Myers RM, Brown M, Li W, Liu XS. Model-based analysis of ChIP-Seq (MACS). Genome Biol. 2008;9:R137. 
88. Zhao T, Tao XY, Feng SL, Wang LY, Hong H, Ma W, Shang GD, Guo SS, He YY Zhou BL, Guan XY. The IncRNA sequences and genome coordinate files. LncRNAin-polyploid-cotton. http://github.com/epi-cotton/LncRNA-in-polyploid-cotton. Accessed 9 Oct 2018.

89. Zhao T, Tao XY, Feng SL, Wang LY, Hong H, Ma W, Shang GD, Guo SS, He YY, Zhou BL, Guan XY. Gossypium arboreum, Gossypium raimondii,

Gossypium hirsutum and Gossypium arboreum x Gossypium raimondii F1 Raw sequence reads. Sequence Read Archive. 2018. https://www.ncbi.nlm. nih.gov/bioproject/?term=PRJNA373801. Accessed 9 Oct 2018.

90. Zhao T, Tao XY, Feng SL, Wang LY, Hong H, Ma W, Shang GD, Guo SS, He YY, Zhou BL, Guan XY. Small RNA sequencing of Gossypium arboreum X Gossypium raimondii F1. Sequence Read Archive. 2018. https://www.ncbi. nlm.nih.gov/bioproject/PRJNA375828/. Accessed 9 Oct 2018.

Ready to submit your research? Choose BMC and benefit from:

- fast, convenient online submission

- thorough peer review by experienced researchers in your field

- rapid publication on acceptance

- support for research data, including large and complex data types

- gold Open Access which fosters wider collaboration and increased citations

- maximum visibility for your research: over $100 \mathrm{M}$ website views per year

At $\mathrm{BMC}$, research is always in progress.

Learn more biomedcentral.com/submissions 